\title{
Maleimide-functionalised PLGA-PEG nanoparticles as mucoadhesive carriers for intravesical drug delivery
}

Article

Accepted Version

Creative Commons: Attribution-Noncommercial-No Derivative Works 4.0

Kaldybekov, D., Filippov, S. K., Radulescu, A. and Khutoryanskiy, V. V. (2019) Maleimide-functionalised PLGAPEG nanoparticles as mucoadhesive carriers for intravesical drug delivery. European Journal of Pharmaceutics and Biopharmaceutics, 143. pp. 24-34. ISSN 0939-6411 doi: https://doi.org/10.1016/j.ejpb.2019.08.007 Available at https://centaur.reading.ac.uk/85583/

It is advisable to refer to the publisher's version if you intend to cite from the work. See Guidance on citing.

To link to this article DOI: http://dx.doi.org/10.1016/j.ejpb.2019.08.007

Publisher: Elsevier

All outputs in CentAUR are protected by Intellectual Property Rights law, including copyright law. Copyright and IPR is retained by the creators or other copyright holders. Terms and conditions for use of this material are defined in the End User Agreement. 


\section{CentAUR}

Central Archive at the University of Reading

Reading's research outputs online 
1 Maleimide-functionalised PLGA-PEG nanoparticles as

\section{2 mucoadhesive carriers for intravesical drug delivery}

3 Daulet B. Kaldybekov ${ }^{a, b}$, Sergey K. Filippov ${ }^{c}$, Aurel Radulescu ${ }^{d}$, Vitaliy V. Khutoryanskiy ${ }^{a, *}$

$4 \quad{ }^{\text {a }}$ School of Pharmacy, University of Reading, Whiteknights, RG6 6AD Reading, United Kingdom

$5 \quad{ }^{\mathrm{b}}$ Department of Chemistry and Chemical Technology, Al-Farabi Kazakh National University, 050040

6 Almaty, Kazakhstan

$7 \quad{ }^{\mathrm{c}}$ Harvard John A. Paulson School of Engineering and Applied Sciences, Harvard University, Cambridge,

$8 \quad$ MA 02138, United States

$9 \quad{ }^{\mathrm{d}}$ Forschungszentrum Jülich GmbH, Jülich Centre for Neutron Science (JCNS) at Heinz Maier-Leibnitz

10 Zentrum (MLZ), Lichtenbergstraße 1, 85748 Garching, Germany

$11 *$ Corresponding author

12 Postal address: School of Pharmacy, University of Reading, Whiteknights, PO Box 224, Reading,

13 RG6 6AD, United Kingdom

14 E-mail address: v.khutoryanskiy@ reading.ac.uk (V.V. Khutoryanskiy)

15 Phone: +44(0) 1183786119

16 Fax: +44(0) 1183784703 


\section{$17 \quad$ Abstract}

18 Low permeability of the urinary bladder epithelium, poor retention of the chemotherapeutic agents due

19 to dilution and periodic urine voiding as well as intermittent catheterisations are the major limitations of

20 intravesical drug delivery used in the treatment of bladder cancer. In this work, maleimide-functionalised

21 poly(lactide-co-glycolide)-block-poly(ethylene glycol) (PLGA-PEG-Mal) nanoparticles were

22 developed. Their physicochemical characteristics, including morphology, architecture and molecular

23 parameters have been investigated by means of dynamic light scattering, transmission electron

24 microscopy and small-angle neutron scattering techniques. It was established that the size of

25 nanoparticles was dependent on the solvent used in their preparation and molecular weight of PEG, for

26 example, $105 \pm 1 \mathrm{~nm}$ and $68 \pm 1 \mathrm{~nm}$ particles were formed from $\mathrm{PLGA}_{20 \mathrm{~K}}-\mathrm{PEG}_{5 \mathrm{~K}}$ in dimethyl sulfoxide

27 and acetone, respectively. PLGA-PEG-Mal nanoparticles were explored as mucoadhesive formulations

28 for drug delivery to the urinary bladder. The retention of fluorescein-loaded nanoparticles on freshly

29 excised lamb bladder mucosa in vitro was evaluated and assessed using a flow-through fluorescence

30 technique and Wash Out ${ }_{50}\left(\mathrm{WO}_{50}\right)$ quantitative method. PLGA-PEG-Mal nanoparticles (NPs) exhibited

31 greater retention on urinary bladder mucosa $\left(\mathrm{WO}_{50}=15 \mathrm{~mL}\right)$ compared to maleimide-free $\mathrm{NPs}\left(\mathrm{WO}_{50}=\right.$

$325 \mathrm{~mL}$ ). The assessment of the biocompatibility of PEG-Mal using the slug mucosal irritation test revealed

33 that these materials are non-irritant to mucosal surfaces.

34 Keywords: urinary bladder, intravesical drug delivery, PLGA-PEG, maleimide, nanoparticles, small35 angle neutron scattering, slug mucosal irritation test, mucoadhesion, Wash Out ${ }_{50}\left(\mathrm{WO}_{50}\right)$. 
Mucoadhesion can be described as the ability of materials to adhere to mucosal membranes for extended periods of time. Transmucosal drug administration is currently employed in ocular (conjunctival and corneal), nasal, pulmonary, oromucosal (buccal and sublingual), gastrointestinal, rectal, vaginal and intravesical drug delivery, and it offers an alternative to injections [1-6].

42 Transmucosal drug delivery provides numerous advantages, including (i) prolonged residence time of a 43 dosage form and high density of blood vessels on mucosal surfaces ensure more efficient and rapid drug 44 absorption; (ii) non-oral drug administration allows avoiding its potential degradation in the stomach and 45 prevents hepatic first-pass metabolism; (iii) ease of drug administration and the possibility for quick 46 termination of a therapy resulting in improved patient compliance; (iv) possibility of targeting particular 47 body sites and tissues due to local administration; and (v) reduced administration frequency. 48 Mucoadhesive dosage forms could be highly beneficial for local drug administration to treat posterior 49 segment diseases of the eye [7,8], neurological disorders (via intranasal administration) $[9,10]$, and 50 genitourinary tract dysfunctions $[11,12]$.

Intravesical drug delivery (IDD) refers to a direct administration of active pharmaceutical

52 ingredients into the urinary bladder using a catheter inserted through the urethra. This technique is 53 generally used to treat bladder-related disorders, such as bladder cancer (BC) and interstitial cystitis [1354 15]. However, the efficiency of this route of drug administration is limited due to dilution and wash out 55 during periodical micturition resulting in poor retention of instilled therapeutic agents. Additionally, the 56 need for frequent catheterisations with potential risks of irritation, inflammatory reactions and infections 57 makes this procedure rather unpleasant for patients [16].

$58 \quad$ Mucoadhesive materials have the potential to improve the efficacy of IDD by prolonging the drug 59 residence in the bladder. First generation (conventional) mucoadhesive materials are traditionally used 60 as matrixes in many formulations for transmucosal drug delivery. These include hydrophilic polymers 61 of natural and synthetic origin, such as chitosan, carbopols and cellulose derivatives [17-21]. The 
62 adhesion of these macromolecules is due to their ability to interact with glycosylaminoglycans/mucins 63 present on mucosal surfaces through non-covalent interactions such as hydrogen bonding, electrostatic 64 attraction and chain entanglement.

65 Andreas Bernkop-Schnürch [22] pioneered the use of thiolated polymers (thiomers) as the second 66 generation of mucoadhesives. Well-established water-soluble polymers were modified with thiol 67 functional groups using different chemical approaches to greatly enhance their mucoadhesive properties 68 [23-25]. A few studies reported the use of thiolated mucoadhesives for IDD. For example, thiol69 functionalised chitosan nanoparticles have been used for IDD in an in vitro study using porcine urinary 70 bladders $[26,27]$. The ability of these thiolated mucoadhesives to stay adhered on mucosal surfaces is 71 due to the formation of inter-disulphide bridges via covalent interaction with cysteine residues present in 72 mucins.

73 Recently, our group has demonstrated that polymeric nanogels [28] and PEGylated liposomes [29] 74 functionalised with maleimide groups exhibited excellent mucoadhesive properties to bovine 75 conjunctival tissues and freshly excised porcine bladder mucosa, respectively. The ability of these 76 formulations to retain on mucosal tissues, evaluated using a flow-through method, was found to be 77 comparable to a well-known mucoadhesive chitosan. The excellent mucoadhesive performance of these 78 novel advanced formulations is attributed to their ability to form covalent linkages with thiol groups 79 present in the mucus via Michael-type addition reactions. Later, other researchers have also reported the 80 development of alginate- and chitosan-based mucoadhesives functionalised with maleimide groups and 81 demonstrated their superior retention/adhesion on/to mucosal surfaces compared to unmodified 82 polysaccharides [30, 31]. All the maleimide-functionalised systems reported so far [28-31] due to their 83 hydrophilic nature were only suitable for formulation of water-soluble drugs. There is also a strong need 84 in the development of mucoadhesive drug delivery systems capable of incorporating poorly-soluble 85 drugs, which will require the use of less polar polymers for their development. 
87 approved by the US Food and Drug Administration, has been widely employed in controlled release 88 formulations and tissue engineering applications due to its safety, low toxicity, low immunogenicity, 89 high cytocompatibility and biodegradability [32]. PLGA-PEG could easily be self-assembled to micelles 90 or nanoparticles by either simple single emulsion (oil-in-water) or double emulsion solvent evaporation 91 (water/oil/water) techniques [33,34]. For instance, Bazylińska et al. [35] described the influence of 92 preparation parameters, the type of polymer and active cargo on the colloidal and biological stability of 93 final nano-carriers for further in vitro and in vivo applications. Pei et al. developed the application of $\mathrm{pH}-$ 94 sensitive intravenous PLGA-PEG formulations to deliver vancomycin in the therapy against intracellular 95 pathogens [36]. PLGA-PEG nanoparticles functionalised with maleimide groups were also recently 96 reported as carriers for drug delivery. Their maleimide-functionalised surface was used for further 97 modification with monoclonal antibody [37] and cell penetrating peptides [38].

In the present work, we report the design of maleimide-functionalised PLGA-PEG nanoparticles

99 as potential mucoadhesive formulations for IDD to urinary bladder and demonstrate that surface 100 maleimide groups could significantly enhance mucoadhesive properties of these materials. Four types of 101 nanoparticles were developed using PLGA-PEG containing 3 and $5 \mathrm{kDa}$ PEG with and without 102 maleimide terminal groups. The structural features of these nanoparticles were studied using small-angle 103 neutron scattering, dynamic light scattering and transmission electron microscopy. The hydrophobic 104 nature of PLGA-based core of the nanoparticles provided an excellent opportunity for formulating poorly 105 water-soluble compound as a model drug. These nanoparticles were loaded with lipophilic fluorescein 106 and their retention on urinary bladder mucosa was studied in vitro. Slug mucosal irritation in vivo test 107 was used to assess the biocompatibility of PEG-Mal present on the surface of these nanoparticles. 


\section{Materials and methods}

\section{$110 \quad$ 2.1. Materials}

111 Four different di-block copolymers of poly(lactide-co-glycolide)-poly(ethylene glycol) ((methoxy112 terminated: Mw 20,000 : 3,000 Da, PDI $1.30\left(\right.$ PLGA $\left._{20 \mathrm{~K}}-\mathrm{PEG}_{3 \mathrm{~K}}\right)$ and $\mathrm{Mw}$ 20,000 : 5,000 Da, PDI 1.65 113 (PLGA $20 \mathrm{~K}-\mathrm{PEG}_{5 \mathrm{~K}}$ ); maleimide-functionalised: Mw 20,000 : 3,400 Da, PDI 2.70 (PLGA20K-PEG $3 \mathrm{~K}-\mathrm{Mal}_{\text {) }}$

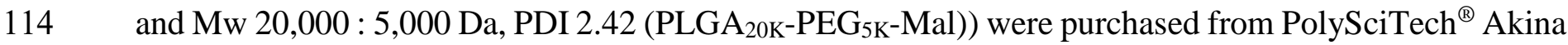
115 Inc. (West Lafayette, IN, USA; catalogue numbers: AK101, AK037, AI109, AI020). Dimethyl sulfoxide 116 (DMSO) was purchased from Fisher Scientific (Loughborough, UK), acetone and deuterium oxide $117\left(\mathrm{D}_{2} \mathrm{O}\right)$, benzalkonium chloride (BAC), poly(ethylene glycol) methyl ether (PEG; average Mn 5,000 Da), 118 methoxypolyethylene glycol maleimide (PEG-Mal; average Mn 5,000 Da) and phosphate buffered saline 119 tablets (PBS) were purchased from Sigma-Aldrich (Gillingham, UK). 6-Maleimidohexanoic acid was 120 purchased from Alfa Aesar (Heysham, UK). Fluorescein was purchased from Acros Organics (Geel, 121 Belgium). All other chemicals were of analytical grade and used as received.

\section{$122 \quad$ 2.2. Preparation of nanoparticles}

123 Empty and fluorescein-loaded PLGA-PEG nanoparticles were prepared via one-step 124 nanoprecipitation (single emulsion) technique followed by dialysis. In brief, $20 \mathrm{mg}$ of polymers and 1 $125 \mathrm{mg}$ fluorescein were dissolved in $1 \mathrm{~mL}$ of DMSO or acetone to form organic phase. The organic phase 126 was then added to $30 \mathrm{~mL}$ of deionised water dropwise under constant stirring to form a colloidal 127 suspension, which was allowed to stir gently for $1 \mathrm{~h}$. Finally, this suspension was purified by dialysis 128 against deionised water (5L; 4 changes) using a dialysis membrane tube (molecular weight cut-off $12-$ $12914 \mathrm{kDa}$; Medicell Membranes Ltd., UK) to remove residual solvent, filtered using $0.22 \mu$ m Minisart $^{\circledR}$ 130 syringe filters and stored in a fridge for further use.

\section{$131 \quad$ 2.3. Particle size and zeta potential analysis}


132 The size of fluorescein-free PLGA-PEG nanoparticles, their polydispersity index (PDI) and zeta133 potential values were determined using dynamic light scattering (DLS) at a scattering angle of $173^{\circ}$ with 134 a Zetasizer Nano-NS (Malvern Instruments, UK). Each nanoparticle dispersion was diluted 100-fold with 135 ultra-purified water. Refractive index of 1.59 and absorbance of 0.01 were used in all measurements. 136 Each sample was analysed three times at $25^{\circ} \mathrm{C}$ and the Z-average mean \pm standard deviation values were 137 calculated.

\subsection{Transmission electron microscopy (TEM)}

139 Nanoparticles were imaged using a JEOL 2100 Plus TEM operated at an acceleration voltage of $140200 \mathrm{kV}$. Specimens were prepared by pipetting a drop of purified nanoparticle suspension (about 0.5 $141 \mathrm{mg} / \mathrm{mL}$ ) onto a parafilm. A glow-discharged holey carbon film-coated 400-mesh copper grid was then 142 placed onto the drop with "carbon" side and left in contact with the sample for $60 \mathrm{sec}$. The excess solution 143 was removed by blotting with a filter paper. Then, a drop of $2 \%(\mathrm{w} / \mathrm{v})$ uranyl acetate (UA) solution was 144 deposited onto the parafilm and the grid remained in contact with "carbon" side with UA for another 60 145 sec. The excess stain was removed by dabbing similarly as above and the sample was left to dry in air 146 prior to TEM characterisation. This sample preparation technique was previously reported to give good 147 quality of images for PLGA-PEG nanoparticles [39].

\section{$148 \quad$ 2.5. Small-Angle Neutron Scattering (SANS) study}

149 PLGA-PEG nanoparticles were prepared as described above. Briefly, $10 \mathrm{mg}$ of polymers were 150 dissolved in $1 \mathrm{~mL}$ of organic solvent (acetone or DMSO). This solution was then added to $20 \mathrm{~mL}$ of $\mathrm{D}_{2} \mathrm{O}$ 151 dropwise under continuous stirring to form suspension of nanoparticles, which was then allowed to stir 152 gently for an additional $20 \mathrm{~min}$. Finally, $2 \mathrm{~mL}$ of aliquot was aspirated from the suspension and dialysed 153 using a Spectra-Por ${ }^{\circledR}$ Float-A-lyzer ${ }^{\circledR}$ G2 dialysis membrane, with a molecular weight cut-off 3.5-5 kDa, 154 against $\mathrm{D}_{2} \mathrm{O}$ to remove residual solvent, filtered using $0.22 \mu \mathrm{m}$ Minisart ${ }^{\circledR}$ syringe filters and stored in a 155 fridge prior to SANS studies. 
157 Measurements were made on a ${ }^{3} \mathrm{He}$ tubes array detector (144 tubes, pixel size $8 \mathrm{~mm}$ ) using a non158 polarised, monochromatic (wavelength $\lambda$ set by a velocity selector) incident neutron beam collimated 159 with rectangular apertures for two sample-to-detector distances, namely 2,8 , and $20 \mathrm{~m}(\lambda=0.6 \mathrm{~nm})$. 160 With this setup, the investigated $q$-range was $0.015 \mathrm{~nm}^{-1}$ to $4.6 \mathrm{~nm}^{-1}$. In all cases, the two-dimensional 161 scattering patterns were isotropic and were azimuthally averaged, resulting in the dependence of the 162 scattered intensity $I_{s}(q)$ on the momentum transfer $q=4 \pi \sin \theta / \lambda$, where $2 \theta$ is the scattering angle. The 163 curves were corrected for background scattering from the empty cell and for detector efficiency. Hellma ${ }^{\circledR}$ 164 Analytics Suprasil ${ }^{\circledR} 300$ high precision quartz cells of 1 and $2 \mathrm{~mm}$ thickness were used for experiments. 165 SANS experiments were performed in $\mathrm{D}_{2} \mathrm{O}$ solution. The $\mathrm{D}_{2} \mathrm{O}$ solution was measured and properly 166 subtracted. The concentration of nanoparticles used in SANS measurements was $0.5 \mathrm{mg} / \mathrm{mL}$.

\subsection{SANS data fitting}

The scattered intensity curves were fitted using the model of sphere with attached Gaussian chain 169 having self-avoiding walk statistics implemented in SASFit software [41] based on the model developed 170 by Pedersen et al. [42] (Fig. 1).

171 The scattering curves in $\mathrm{D}_{2} \mathrm{O}$ could be fitted using the following function:

$172 \quad I(q)=P_{s g c}(q) S(q)$

173 We assume that $S(q)=1$ due to low concentration of nanoparticles in solution, $1 \mathrm{mg} / \mathrm{mL}$. The 174 overall scattering intensity of the sphere with attached Gaussian chain written as:

$$
P_{\text {sgc }}=N_{\text {agg }}^{2} \beta_{\text {core }}^{2} P_{\text {core }}(q)+N_{\text {agg }} \beta_{\text {brush }}^{2} P_{\text {brush }}(q)+2 N_{\text {agg }}^{2} \beta_{\text {core }} \beta_{\text {brush }} S_{\text {brush-core }}(q)+
$$

$$
N_{\text {agg }}\left(N_{\text {agg }}-1\right) \beta_{\text {brush }}^{2} S_{\text {brush-brush }}(q)
$$


177 where $N_{\text {agg }}^{2} \beta_{\text {core }}^{2} P_{\text {core }}(q)$ is self-correlation term of the core; $N_{a g g} \beta_{b r u s h}^{2} P_{b r u s h}(q)$ is self-correlation 178 term of the chains; $2 N_{\text {agg }}^{2} \beta_{\text {core }} \beta_{\text {brush }} S_{\text {brush-core }}(q)$ is the cross-term between the core and chains and $179 N_{a g g}\left(N_{a g g}-1\right) \beta_{\text {brush }}^{2} S_{\text {brush-brush }}(q)$ is the cross-term between different chains. $N_{a g g}$ is the 180 aggregation number of polymers forming the nanoparticle per surface area, $\beta_{b r u s h}=V_{b r u s h}\left(\eta_{b r u s h}-\right.$ $\left.181 \eta_{\text {solv }}\right)$ and $\beta_{\text {core }}=V_{\text {core }}\left(\eta_{\text {core }}-\eta_{\text {solv }}\right)$ are the excess scattering lengths of a block in the corona and in 182 the core, respectively. $V_{\text {brush }}$ and $V_{\text {core }}$ are the total volume of a block in the corona and in the core, 183 respectively. $\eta_{\text {brush }}$ and $\eta_{\text {core }}$ are the corresponding scattering length densities (SLDs). $P_{\text {core }}(q)$ is 184 scattering of spherical core

$185 \quad P_{\text {core }}\left(q, R_{\text {core }}\right)=3 \frac{\left(\sin \left(q R_{\text {core }}\right)-q R_{\text {core }} \cos \left(q R_{\text {core }}\right)\right)}{\left(q R_{\text {core }}\right)^{3}}$

188 where $x=R_{\text {gchain }}^{2} q^{2} ; R_{\text {gchain }}$ is the gyration radius of a polymer chain.

The contribution of cross term between core and chains which form brush of wormlike micelle is calculated using equation:

$$
S_{\text {brush-core }}\left(q, R_{\text {core }}, R_{\text {gchain }}, d\right)=\psi\left(q R_{\text {gchain }}\right) P_{\text {core }}\left(q, R_{\text {core }}\right) \frac{\sin \left(q\left[R_{\text {core }}+d R_{\text {gchain }}\right]\right)}{q\left[R_{\text {core }}+d R_{\text {gchain }}\right]}
$$

192 where $\psi\left(q R_{\text {gchain }}\right)=\frac{1-\exp (-x)}{x}$ is the form factor amplitude of the chain.

The contribution of cross term between chains is calculated using equation:

$$
S_{\text {brush-brush }}\left(q, R_{\text {core }}, R_{\text {gchain }}, d\right)=\psi^{2}\left(q R_{\text {gchain }}\right)\left[\frac{\sin \left(q\left[R_{\text {core }}+d R_{\text {gchain }}\right]\right)}{q\left[R_{\text {core }}+d R_{\text {gchain }}\right]}\right]^{2}
$$


195 where $d$ is parameter that accounts for non-penetration of the chains into the core and should be 196 mimicked by $d \sim 1$ for $R_{\text {core }} \gg R_{\text {gchain }}$.

197 The model has the following fitting parameters: $R_{\text {core }}-$ core radius; $V_{\text {core }}-$ molecular volume of 198 single block unit in the micellar core; $V_{b r u s h}-$ molecular volume of single block unit in the micellar 199 corona; $\eta_{\text {core }}$ - scattering length density of spherical core; $\eta_{b r u s h}-$ scattering length density of the block 200 unit in the corona; $\eta_{\text {solv }}$ - scattering length density of solvent; $R_{\text {gchain }}$ - gyration radius of polymer 201 chains in the corona; $L$ - contour length of polymer chain, $b$ - Kuhn segment length.

202 Excess scattering lengths of solvent and polymeric shell, $V_{c o r e}, V_{b r u s h}$, contour length of polymer 203 chain, Kuhn segment length were known from literature data and polymer composition and were chosen 204 to be fixed during the fitting procedure.

205 To account for nanoparticles polydispersity, a Schulz-Zimm distribution of $R_{\text {core }}$ with 206 polydispersity parameter $\sigma$ was included in the following way:

$207 \quad S Z=\frac{R_{\text {core }}^{Z}}{\Gamma(Z+1)}\left(\frac{Z+1}{<R_{\text {core }}>}\right)^{Z+1} \exp \left[-\frac{(Z+1) R_{\text {core }}}{<R_{\text {core }}>}\right]$

$208 \quad$ where $Z=\frac{1}{\sigma^{2}}-1$

209 The gyration radius $R_{g}$ of nanoparticles was calculated from Guinier regime to evaluate the overall $210 \quad$ size of nanoparticles.

\section{$211 \quad$ 2.7. Encapsulation efficiency and loading capacity}

Amicon ${ }^{\circledR}$ Ultra-0.5 Ultracel-3 centrifugal filter unit with a molecular weight cut-off $10 \mathrm{kDa}$ was

213 used in these experiments. Each centrifugal filter device was pre-rinsed with PBS (500 $\mu \mathrm{L})$ at 13,000

$214 \operatorname{rpm}(7558 \times \mathrm{g})$ for $30 \mathrm{~min}$ prior to further use. The dispersion of PLGA-PEG nanoparticles $(500 \mu \mathrm{L})$ was

215 placed in an ultrafiltration tube and centrifuged at $4{ }^{\circ} \mathrm{C}$ at $13,000 \mathrm{rpm}(7558 \times \mathrm{g})$ for $30 \mathrm{~min}$. This loading 
216 step was repeated twice. The filtrate was discarded and the retentate was washed with $250 \mu \mathrm{L}$ of PBS by

217 further centrifugation at $4{ }^{\circ} \mathrm{C}$ at $13,000 \mathrm{rpm}(7558 \times \mathrm{g})$ for $20 \mathrm{~min}$. The filtrate was withdrawn again and 218 the fluorescein-loaded PLGA-PEG nanoparticles in the retentate were then mixed with $100 \mu \mathrm{L}$ DMSO 219 (left for $6 \mathrm{~h}$ in a fridge to dissolve the nanoparticles and model drug) and spun at $4{ }^{\circ} \mathrm{C}$ at $13,000 \mathrm{rpm}$ $220(7558 \times \mathrm{g})$ for $10 \mathrm{~min}$. The amount of free fluorescein in the supernatant was quantified using a Varian 221 Cary Eclipse fluorescence spectrophotometer at $\lambda_{\text {excitation }}$ and $\lambda_{\text {emission }}=460$ and $513 \mathrm{~nm}$, respectively, 222 and the encapsulation efficiency (EE\%) and loading capacity (LC\%) were determined using the 223 following formulae:

$224 \quad E E \%=\frac{C}{C_{i}} \times 100$

$225 \quad L C \%=\frac{C}{\text { Total weight of NPs }} \times 100$

226 where $C$ is the amount of fluorescein encapsulated in the nanoparticles (NPs), and $C_{i}$ is the initial amount

227 of fluorescein. A standard curve was generated by plotting the fluorescence intensities from different 228 concentrations of the model drug and used to calculate EE\% and LC\% can be found in Supplementary 229 Information (Fig. S1).

\section{$230 \quad$ 2.8. Toxicology: slug mucosal irritation test}

231 The slug mucosal irritation test (SMIT) was carried out according to our previously published 232 report [43]. Arion lusitanicus slugs were collected locally in Harris Garden (Reading, UK) and were 233 housed in specially designed plastic containers and fed with lettuce, cabbage, carrots and cucumber. Each 234 slug's body lining was carefully examined and only slugs showing no evidence of macroscopic injuries 235 with clear tubercles and a foot surface were used for testing purposes. Slugs weighing between 15 and $23623 \mathrm{~g}$ were isolated from the culture and were kept individually in $1.5 \mathrm{~L}$ glass beakers lined with a paper 
237 towel soaked with $20 \mathrm{~mL}$ of PBS solution and left at room temperature for $48 \mathrm{~h}$ before the start of an 238 experiment. All beakers were covered with a cling film pierced with tiny holes in order to allow air 239 exchange. Each slug was individually weighed before the experiment and then placed in $90 \mathrm{~mm}$ Petri

240 dishes lined with Whatman ${ }^{\mathrm{TM}}$ filter paper moistened with either positive/negative controls $(2 \mathrm{~mL}$ of $1 \%$ 241 BAC prepared in PBS and $2 \mathrm{~mL}$ of PBS solution, respectively) or $2 \mathrm{~mL}$ of each test materials (PEG, 242 PEG-Mal and MHA) with the following concentrations: $0.00003 ; 0.0003 ; 0.003$ and 0.03 mmol prepared 243 in PBS. After 60 min contact period slugs were taken out, rinsed with $10 \mathrm{~mL}$ of PBS, gently wiped with 244 the paper towel and then reweighed. The percentage of mucus production (MP\%) was estimated as a slug 245 body weight loss and evaluated using the following equation:

$246 \quad M P \%=\frac{\left(m_{b}-m_{a}\right)}{m_{b}} \times 100$

247 where $m_{b}$ and $m_{a}$ are the weights of a slug before and after experiment, respectively. Each experiment 248 was repeated 7 times using different slugs and the results were evaluated statistically, calculating the 249 mean \pm standard deviation values.

\section{$250 \quad$ 2.9. Preparation of artificial urine solution}

Artificial urine solution was prepared according to the previously reported protocol [44]. Briefly, 252 the following components were dissolved in deionised water by stirring overnight at room temperature, 253 before making the total volume to $2000 \mathrm{~mL}$ : urea $(24.27 \mathrm{~g})$, uric acid $(0.34 \mathrm{~g})$, creatinine $(0.90 \mathrm{~g})$, sodium 254 citrate dihydrate $\left(\mathrm{Na}_{3} \mathrm{C}_{6} \mathrm{H}_{5} \mathrm{O}_{7} \cdot 2 \mathrm{H}_{2} \mathrm{O}, 2.97 \mathrm{~g}\right)$, sodium chloride $(\mathrm{NaCl}, 6.34 \mathrm{~g})$, potassium chloride $(\mathrm{KCl}$, $2554.50 \mathrm{~g})$, ammonium chloride $\left(\mathrm{NH}_{4} \mathrm{Cl}, 1.61 \mathrm{~g}\right)$, calcium chloride dihydrate $\left(\mathrm{CaCl}_{2} \cdot 2 \mathrm{H}_{2} \mathrm{O}, 0.89 \mathrm{~g}\right)$, 256 magnesium sulfate heptahydrate $\left(\mathrm{MgSO}_{4} \cdot 7 \mathrm{H}_{2} \mathrm{O}, 1.00 \mathrm{~g}\right)$, sodium bicarbonate $\left(\mathrm{NaHCO}_{3}, 0.34 \mathrm{~g}\right)$, sodium 257 oxalate $\left(\mathrm{Na}_{2} \mathrm{C}_{2} \mathrm{O}_{4}, 0.03 \mathrm{~g}\right)$, sodium sulphate $\left(\mathrm{Na}_{2} \mathrm{SO}_{4}, 2.58 \mathrm{~g}\right)$, sodium phosphate monobasic monohydrate 
$258\left(\mathrm{NaH}_{2} \mathrm{PO}_{4} \cdot \mathrm{H}_{2} \mathrm{O}, 1.00 \mathrm{~g}\right)$, and sodium phosphate dibasic $\left(\mathrm{Na}_{2} \mathrm{HPO}_{4}, 0.11 \mathrm{~g}\right)$. The artificial urine solution $259 \quad$ (pH 6.40) was kept at $37^{\circ} \mathrm{C}$ throughout the experiments.

\subsection{In vitro retention studies on lamb urinary bladder mucosa}

The retention of PLGA-PEG nanoparticles on lamb urinary bladder tissues in vitro was determined using a protocol previously described by our group with minor modifications [29]. Lamb bladder tissues were received from P.C. Turner Abattoirs (Farnborough, UK) immediately after animal slaughter, packed, frozen and transported in an insulated plastic container. The tissues were subsequently thawed upon arrival and carefully excised to yield approximately $2 \times 3 \mathrm{~cm}$ sections, avoiding contact with the internal mucosa, which were then used in the experiments. The dissected bladder tissue was mounted on a glass slide with mucosal side facing upward and rinsed with $3 \mathrm{~mL}$ of artificial urine (AU; $\mathrm{pH} 6.40$ ) solution. Experiments were performed with the bladder tissues maintained at $37{ }^{\circ} \mathrm{C}$ in an incubator. Aliquots $(200 \mu \mathrm{L})$ from fluorescein-loaded PLGA-PEG nanoparticle stock solutions were withdrawn and deposited onto a mucosal surface and rinsed with $\mathrm{AU}$ at a constant flow rate of $2 \mathrm{~mL} / \mathrm{min}$ using a syringe pump (total washing time was $50 \mathrm{~min}$ ). Fluorescence images of a bladder tissue were taken using Leica MZ10F stereo-microscope (Leica Microsystems, UK) with Leica DFC3000G digital camera at 1.6x magnification with $30 \mathrm{~ms}$ exposure time (gain 2.0x), fitted with a GFP filter. The microscopy images were then analysed with ImageJ software by measuring the pixel intensity after each irrigation with AU. The pixel intensity of the blank samples (bladder mucosa without test material) were subtracted from each measurement and data are converted into numbers. All measurements were conducted in triplicate.

Evaluation of formulations retention on the mucosa in vitro was quantified through $\mathrm{WO}_{50}$ values, which represent the volume of a biological fluid necessary to wash out $50 \%$ of a mucoadhesive excipient from a substrate [45]. $\mathrm{WO}_{50}$ values of test materials were calculated via extrapolation of the average wash-off profiles to $50 \%$ using polynomial fitting (5th order) and Wolfram Alpha (a computational knowledge engine). 


\subsection{Statistical analysis}

Statistical analysis of data, i.e. mean values \pm standard deviations were calculated and assessed for significance using two-tailed Student's $t$-test and a one-way analysis of variance (ANOVA) followed by Bonferoni post hoc test using GraphPad Prism software (version 7.0), where $p<0.05$ was fixed as the statistical significance criterion.

\section{Results and discussion}

\subsection{Preparation and characterisation of nanoparticles}

PLGA-PEG nanoparticles, with and without fluorescein, were formulated using single emulsion 290 method and precipitated from acetone and DMSO. The detailed preparation procedure of nanoparticles 291 is illustrated in Fig. 2. The average mean diameter of all PLGA-PEG nanoparticles precipitated from 292 DMSO remained $\sim 100 \pm 1 \mathrm{~nm}$ on average regardless of the molecular weight of PEG moiety in block 293 copolymers, whereas the nanoparticles precipitated from acetone displayed smaller values of $65 \pm 1 \mathrm{~nm}$ 294 and $80 \pm 1 \mathrm{~nm}$ for PLGA-PEG and PLGA-PEG-Mal, respectively. We believe that the difference in the 295 particle sizes might depend on the dielectric constant of organic solvent (acetone, $\varepsilon=21$ and DMSO, $\varepsilon$ $296=47)$ and their miscibility with water during formation of nanoparticles in aqueous phase [46]. It is also 297 interesting to compare our results with the study reported by Yang et al. [47], who prepared PLGA-PEG 298 nanoparticles from acetone, acetonitrile and tetrahydrofuran. They reported the solvent effect on the 299 particle size with formation of $154 \pm 3,134 \pm 2$ and $186 \pm 4 \mathrm{~nm}$ of nanoparticles from acetone, acetonitrile 300 and tetrahydrofuran, respectively. Our nanoparticles prepared from acetone are much smaller $(65 \pm 1$ $301 \mathrm{~nm}$ ), which shows the strong influence of other factors, including individual block molecular weights 302 and particle preparation techniques.

303 All nanoparticles prepared in the present study showed negative zeta-potential greater than $-21 \mathrm{mV}$ 304 and had low polydispersity of less than 0.20 , indicating the presence of a homogeneous population with 
a narrow size distribution (Fig. 3). The physicochemical characteristics of PLGA-PEG nanoparticles are 306 summarised in Table 1.

Fluorescein was used as a model drug to demonstrate the potential application of PLGA-PEG nanoparticles in drug delivery to urinary bladder. Fluorescein (partition coefficient, $\log \mathrm{P}=3.35$, [48]) was loaded into the nanoparticles by first preparing the model drug solution in the organic solvent followed by dissolution of polymers and further nanoprecipitation in deionised water.

The size and morphology of nanoparticles were further confirmed by TEM analysis and microphotographs are displayed in Fig. 4. Uranyl acetate was used as a negative staining to achieve

313 reasonable contrast. TEM analysis revealed the formation of homogeneous vehicles with well-dispersed 314 spherically shaped core and shell structure of nanoparticles and the results are in good agreement with 315 the data obtained by DLS measurements (Table 1).

\subsection{Nanoparticle structure}

Small-angle neutron scattering (SANS) was used to probe the nanoparticle architecture and 318 determine their molecular parameters. The use of SANS allows to get more information about small 319 particles compared to DLS method. The scattering intensity $I(q)$ curves for all nanoparticle solutions are 320 presented in Fig. 5A and 5B. Several issues should be noted from the inspection of these scattering 321 curves. First, all curves have monotonous behaviour with $q$; no additional maxima are observed. It is a 322 typical manifestation of moderate or high polydispersity for compact objects. Second, all curves are 323 similar in the middle and high $q$ ranges that implies similarity of all nanoparticles from inside 324 disregarding preparation way and used solvent. The only difference between the scattering curves is 325 visible in low $q$ range that is probably related to the difference in the aggregation number. To have a 326 deeper information, the gyration radius values of nanoparticles in solution were calculated using Guinier 327 approximation (Table 2). Comparison of gyration and hydrodynamic radii reveals that these two 328 parameters are clearly correlated. In agreement with DLS data, the gyration radius value for PLGA-PEG 
and PLGA-PEG-Mal nanoparticles precipitated from DMSO is nearly the same and insensitive to the

330 PEG molecular weight to the presence of maleimide groups (Table 2). In contrast, the gyration radius

331 value of nanoparticles precipitated from acetone is higher for longer length of a PEG chain. The ratio of

332 gyration and hydrodynamic radii $R_{g} / R_{h}$ have been known as the $\rho$ parameter that is sensitive to the 333 architecture of nano-objects. It provides model-independent clue on spatial arrangement of a scattering 334 object [49-53], its theoretical value is known for some simple models such as a hard sphere $(\rho=$ 335 0.775), Gaussian chain in $\Theta$-solvent $(\rho=1.5)$, long rods $(\rho>2.0)$. The $\rho$ parameter value calculated 336 for PLGA-PEG and PLGA-PEG-Mal nanoparticles precipitated from DMSO undoubtedly pointing out 337 on a spherical symmetry and compact structure in agreement with TEM results presented above. The 338 change from DMSO to acetone results in the formation of nanoparticles with more loose structure since 339 they have higher $\rho$ parameter value. Increasing of PEG molecular weight leads to increase in $\rho$ value 340 highlighting the enhanced branching structure for $5 \mathrm{kDa}$ PEG nanoparticles made from acetone. We have 341 to mention here that some of these systems have $\rho$ value that is below the lowest possible theoretical 342 limit, 0.775. Nevertheless, low values were already reported in a variety of publications. The $\rho$ parameter 343 fluctuates around 0.8 , an average value for all types of nanoparticles justifying the choice of the fitting 344 model described in Experimental Section.

345 The scattering curves obtained for the $\mathrm{D}_{2} \mathrm{O}$ solutions can be well fitted with the "sphere with 346 attached Gaussian chain having self-avoiding walk statistics" model, assuming a Schulz-Zimm 347 distribution for core radius. The calculated structural parameters are presented in Table 2.

348 The obtained value of the core radii and shell thickness are in the range of $12-24 \mathrm{~nm}$, which is in 349 agreement with the $R_{h}$ as observed in the DLS measurements and TEM. In all series of nanoparticles 350 prepared by precipitation from acetone and DMSO, $R_{\text {gchain }}$ increases with increase in PEG molecular 351 weight (Table 2), whereas $R_{\text {core }}$ is much less sensitive to PEG chain length. 
352 Our results corroborate with SANS studies of PLGA-PEG based block copolymers published 353 previously [47,54-57] keeping in mind the difference in composition, molecular weights and methods of 354 preparation. For example, PEO-PLGA-PEO [55] and PLGA-PEG-PLGA [56] triblock copolymers were 355 investigated using SANS. Apart from the difference in composition, triblock $v s$ diblock, the copolymers 356 reported in these papers were different on other important aspects. The molecular weight, Mn, of PLGA 357 block was much lower than the one in our case, 3,500 g/moL [55] and 1,170 g/moL [56], respectively. 358 In both cases solutions were prepared by direct dissolving in $\mathrm{D}_{2} \mathrm{O}$ in contrast with solvent exchange 359 method exploited in the present paper. Additionally, structure factor was involved in the fitting procedure 360 since the triblock copolymer solution were in semi-diluted regime, 24 and 20 wt.\% for PEO-PLGA-PEO 361 and PLGA-PEG-PLGA, respectively. Applying the Percus-Yevick (PY) hard-sphere model to describe 362 the structure factor and Global Indirect Fourier Transformation (GIFT) to describe the form factor of the 363 micelles composed of PEO-PLGA-PEO at $30{ }^{\circ} \mathrm{C}$, the authors obtained the following values for the radii 364 of a core and shell, 5.9 and $2.5 \mathrm{~nm}$, respectively [55]. The presence of a shell was also reported by 365 Khorshid et al for flower-like micelles composed by PLGA-PEG-PLGA triblock copolymers [56]. SANS 366 method successfully disseminates the internal structure of nanoparticles. The SANS experiments give a 367 clear evidence of asymmetric ellipsoid nanoparticles for the copolymer with the PEG block $(\mathrm{Mn}=1,000$ $368 \mathrm{~g} / \mathrm{moL}$ ), whereas spherical micelles with core-shell topology is observed for the polymer with longer 369 PEG central block $(\mathrm{Mn}=1,500 \mathrm{~g} / \mathrm{moL})$. The core-shell cylinder and spherical shell models were utilised 370 to fit the SANS data [56]. Unlike the PEO-PLGA-PEO system, both PLGA-PEG-PLGA copolymers 371 were studied not only in semi-diluted but also for diluted concentration, $1 \mathrm{wt} \%$. The core and shell radii 372 for $\mathrm{PLGA}_{1170}-\mathrm{PEG}_{1500}-\mathrm{PLGA}_{1170}$ were $5.0 \mathrm{~nm}$ and these values are much lower in comparison with the 373 data reported in our manuscript, $\mathrm{R}_{\text {core }}$ and $\mathrm{R}_{\text {gchain, }}$ Table 2, that is not surprising keeping in mind much 374 higher molecular weights for PLGA and PEG of our copolymers.

375 Interesting contrast variation SANS study was performed for laponite/PEG1.0k-PLGA0.8k and 376 laponite/PEG1.0k-PLGA1.6k nanocomposites in a broad range of $\mathrm{D}_{2} \mathrm{O} / \mathrm{H}_{2} \mathrm{O}$ mixture concentrations [57]. 
377 It was found that for $66 \%$ of $\mathrm{D}_{2} \mathrm{O}$, the scattering from laponite is matched to the scattering of solvent. 378 The combination of core-shell spherical model in combination with Debay form factor was used for the 379 fitting the scattering profile from laponite free nanoparticles made of PEG-PLGA copolymers. The radius 380 of a core and the micelles was found 1.6 and $4.7 \mathrm{~nm}$, respectively, giving the shell thickness value to be 381 of $3.1 \mathrm{~nm}$ [57]. We note here that the model used in our work is very close to the one reported in [57].

382 Two SANS studies that are the most relevant to our paper deal with nanoparticles composed from PLGA383 PEG [47] and PLA-PEG [54] diblock copolymers. In both papers, nanoparticles were prepared by solvent 384 exchange method. The spherical model with a polydisperse core of constant scattering density and 385 diffusive shell was applied for the fitting of PLA-PEG copolymers [54]. The fitting data reported for the 386 acetone as the organic solvent and copolymer with similar composition $\mathrm{PLA}_{15600}-\mathrm{PEG}_{5000}$ are in perfect 387 agreement with our results for $\mathrm{PLGA}_{20 \mathrm{~K}}-\mathrm{PEG}_{5 \mathrm{~K}}$ (Table 2). The mean core radius of the nanoparticles 388 made of PLA $_{15600}-\mathrm{PEG}_{5000}$ is $11 \mathrm{~nm}$, which correlates with our results, $12 \mathrm{~nm}$. The Schultz-Zimm 389 poldispersity values are almost identical, 0.27 [54] vs 0.21 in our study. The thickness of the PEG shell 390 of $6.7 \mathrm{~nm}$ for $\mathrm{PLA}_{15600}-\mathrm{PEG}_{5000}$ also agrees well with our data for $\mathrm{PLGA}_{20 \mathrm{~K}}-\mathrm{PEG}_{5 \mathrm{~K}}$ that was found to be $391 \quad 6.9 \mathrm{~nm}$.

392 Surprisingly, the similar systems measured recently show clearly distinctive results [47]. The 393 PLGA-PEG nanoparticles prepared by solvent exchange method presumably composed from micelles 394 that form a fractal structure inside of a nanoparticle. One of the plausible explanations could be the 395 existence of some residual reactive groups on PLGA-PEG copolymers reported in [47]. Such reactive 396 groups might form bridges between PLGA-PEG micelles.

397 The choice of the solvent used for precipitation has the greatest impact on nanoparticles structure. 398 Interestingly, nanoparticles dissolved and precipitated from acetone have not only a smaller core but also 399 lower $R_{\text {gchain }}$ value in comparison with the NPs precipitated from DMSO. This is a rather unpredicted 400 result that might indicate a different local structure of the interface between PLGA core and PEG brush 401 and different conformation of PEG chains. Indeed, the gyration radius of a PEG chain is twice lower for 
402 NPs precipitated from acetone. Such striking difference could only be explained by compaction of PEG 403 chains on the surface of nanoparticles precipitated from acetone and, in reverse, fully stretched PEG 404 conformation in case of NPs made from DMSO. This hypothesis is indirectly supported by another 405 peculiar feature observed from the SANS results fitting: the presence of maleimide terminal groups 406 increases the $R_{\text {gchain }}$ for DMSO series, and in reverse, decreases $R_{\text {gchain }}$ for NPs precipitated from 407 acetone (Table 2). We hypothesize that NPs prepared from DMSO have a narrow interface between 408 PLGA and PEG blocks, whereas precipitation from acetone somehow facilitates the formation of the 409 broad interface layer where PLGA and PEG coexist. Inevitably, bulky maleimide groups attached to the 410 dangling PEG chains increase the gyration radius of PEG for NPs precipitated from DMSO. For NPs 411 precipitated from acetone we might expect that some of Mal groups, due to hydrophobic nature of 412 maleimide, will have tendency to incorporate into smooth PLGA-PEG interface (see inserts in Fig. 5).

413 Provided that the nanoparticles precipitated from DMSO have the greatest presence of active maleimide 414 groups on their surface, all subsequent experiments were performed with these samples.

$415 \quad$ 3.3. Mucosal irritancy

416 The information about the biocompatibility and toxicological characteristics of maleimide417 containing materials is currently lacking in the literature. Previously, the biocompatibility of 418 polysaccharides functionalised with maleimide groups was evaluated only by Shtenberg et al. [30] using 419 normal human dermal fibroblasts and by Sahatsapan et al. [31] using human gingival fibroblast cells.

420 The slug mucosal irritation test (SMIT) was developed by Adriaens et al. [58,59] to evaluate the 421 mucosal irritancy potential of different formulations and active ingredients. It uses terrestrial slug species, 422 which are considered to have limited sentience and so are not protected by regulations covering animal 423 experiments [58]. Normally, slugs release mucus to aid their locomotion. They also produce mucus and 424 lose body weight when in contact with irritating substrates. When their mucosal tissue is damaged the 425 slugs release additional proteins and enzymes. This indication allows for quantifiable outcomes as for 
irritants to be classified as non-irritating, mild or severely irritating. Generally, mild irritants cause an

427 increase in mucus production, whereas severe irritants result in tissue erosion and more mucus release in 428 addition to increased production [60].

Previously we reported the use of SMIT for assessing mucosal irritancy of 2430 hydroxyethylmethacrylate and 2-hydroxyethylacrylate based random copolymers of different 431 compositions as well as blend films based on poly(acrylic acid) and methylcellulose using Limax flavus 432 and Arion lusitanicus slugs [43,61]. In the present work, we have adopted the same methodology and 433 used Arion lusitanicus slugs to evaluate the ability of model compounds PEG, PEG-Mal and 6434 maleimidohexanoic acid (MHA) to cause mucosal irritation. Fig. 6 presents the results on mucus 435 production by slugs exposed to filter paper surfaces moistened with solutions of PEG, PEG-Mal and 436 MHA of various concentrations prepared in PBS as well as positive and negative controls. In experiments 437 with $1 \%$ solution of BAC in PBS ( $\mathrm{pH} 7.50)$, used as a positive control, slugs experienced a severe 438 discomfort, producing approximately $38 \pm 7 \%$ of yellow mucus, whereas slugs exposed to PBS (used as 439 a negative control, $\mathrm{pH}$ 7.74) did show a low level of mucus production of $5 \pm 1 \%$. These data are in good 440 agreement with our previous studies [43,61]. A significant variability of the data obtained from 441 experiments with positive control is explained by slugs' increased activity and tendency to escape a 442 contact with an irritant chemical. In all experiments with negative control and polymeric excipients slugs 443 secreted colourless mucus, which is the first demonstration of their reasonably good biocompatibility.

444 The concentration of test materials of $0.0003 \mathrm{mmol}$, used to prepare PLGA-PEG nanoparticles in 445 dispersion, was chosen as a reference for the preparation of liquid formulations with model maleimide446 containing compounds (MHA and PEG-Mal). Filter paper soaked with 0.03 mmol MHA (pH 4.34) 447 displayed significantly higher irritancy $(p<0.001)$ compared to PEG-Mal $(0.03$ mmol; MP $6 \pm 2 \%$; $\mathrm{pH}$ 448 6.92) with the level of mucus production reaching $21 \pm 7 \%$ and confirming the ability of MHA to irritate 449 mucosal epithelia due to, mainly, its acidic nature (Fig. 6). Additionally, slugs exposed to positive control 450 exhibited an extreme discomfort $(p<0.0001)$ compared to slugs in response to contact with $0.0003 \mathrm{mmol}$ 
451 PEG-Mal. However, filter paper soaked with increasing concentrations of PEG and PEG-Mal 452 demonstrated low irritation potential as no significant differences $(p>0.5)$ were observed between these 453 materials and the negative control. Fig. S2 and Fig. S3 in Supplementary Information provide the detailed 454 schematic illustration of SMIT test and the photographs of mucus production by Arion lusitanicus slugs 455 exposed to various test chemicals, respectively. Safety, non-toxicity, and biocompatibility of PEG-Mal 456 allows us to conclude that PLGA-PEG-Mal nanoparticles should not cause irritation in the bladder lining.

457 The non-irritant nature of maleimide-functionalised materials established in this study is also in good 458 agreement with the data reported by Shtenberg et al. [30], who reported that alginate modified with 459 maleimide-terminated PEG is non-toxic to normal human dermal fibroblasts. The maleimide460 functionalised chitosan was also reported by Sahatsapan et al. [31] to be non-toxic to gingival fibroblast 461 cells (HGF) up to $1000 \mu \mathrm{g} / \mathrm{mL}$ of polymer in solution.

\subsection{Mucoadhesion studies}

The retention properties of fluorescein-loaded PLGA-PEG and PLGA-PEG-Mal nanoparticles on

464 lamb urinary bladder mucosa were evaluated using a flow-through method with fluorescent detection 465 using the methodology described in our previously published paper [29]. Fig. 7 shows exemplary 466 fluorescent images of the retention of PLGA-PEG and PLGA-PEG-Mal nanoparticle dispersions on 467 urinary bladder mucosa irrigated with AU. After analysis of the fluorescent images using ImageJ

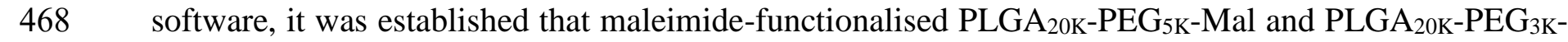
469 Mal nanoparticles exhibited greater mucoadhesive properties compared to unmodified counterparts ( $p<$ 470 0.001), confirming that PLGA-PEG-Mal nanoparticles adhere well to the bladder mucosa by forming 471 covalent linkages with thiol groups present in mucin layer of the bladder epithelium (Fig. 8). Such 472 selective binding leads to increased urothelium cell uptake and potentially improved drug bioavailability 473 within the bladder wall. The nanoparticles remained on the bladder mucosa even after 50 min of washing 474 with a cumulative AU volume of $100 \mathrm{~mL}$. This is in good agreement with our previous findings [28,29], 
475 therefore the results of retention study confirm the mucoadhesive properties of maleimide-functionalised 476 PLGA-PEG nanoparticles, which could also be used as potential mucoadhesive drug carriers in IDD to 477 urinary bladder.

478 Previously we have introduced a novel quantitative method of evaluating and comparing the 479 retention efficacy of liquid formulations on mucosal membranes through the use of $\mathrm{WO}_{50}$ values, which 480 represent the volume of a biological fluid necessary to wash out $50 \%$ of the mucoadhesive test material 481 from a substrate [45]. In this study, we employed the same method and calculated $\mathrm{WO}_{50}$ values by 482 analysing individual wash-off profiles for each nanoparticle suspensions and the results are presented in 483 Table 1. By comparing these values for different PLGA-PEG nanoparticles used in this study, it is clear 484 that $\mathrm{PLGA}_{20 \mathrm{~K}}-\mathrm{PEG}_{5 \mathrm{~K}}-\mathrm{Mal}$ have greater retention on lamb bladder mucosa $\left(\mathrm{WO}_{50}=15 \mathrm{~mL}, \mathrm{R}^{2}=1\right)$ 485 compared to unmodified PLGA $20 \mathrm{~K}-\mathrm{PEG}_{5 \mathrm{~K}}\left(\mathrm{WO}_{50}=5 \mathrm{~mL}, \mathrm{R}^{2}=0.9872\right)$.

\section{$486 \quad 4 . \quad$ Conclusions}

Four different types of PLGA-PEG nanoparticles were prepared and evaluated in this study for 488 their physicochemical characteristics and retention on urinary bladder mucosa. The nanoparticles 489 decorated with maleimide functional groups demonstrated greater retention on the bladder mucosa in 490 vitro due to their ability to form covalent linkages with thiol groups of glycoproteins expressed on the 491 bladder epithelial membrane. The biocompatibility of PEG-Mal was confirmed by SMIT assay 492 experiments.

493 The development of mucoadhesive drug delivery systems that could improve drug residence in the 494 bladder will clearly be beneficial for the treatment of bladder cancer. However, all existing strategies to 495 develop mucoadhesive materials do not provide dosage forms that could selectively bind to cancer cells 496 without adhesion to healthy epithelial cells. The development of systems that could target cancer cells in 497 the bladder is one of the challenges for future research and development in this area. 
The maleimide-functionalised PLGA-PEG mucoadhesive nanoparticles developed in this work 499 could potentially be considered as a platform technology that could be used for drug delivery not only to 500 the bladder but also to other mucosal routes of drug administration. Further research will be focused on 501 biodegradation of these nanoparticles and studies of anticancer drugs encapsulation and release.

$502 \quad$ Acknowledgements

503 The authors gratefully acknowledge the British Council Newton - Al-Farabi Partnership 504 Programme, the Researcher Links Post-Doctoral Mobility Grant (No. 216046068) for financial support 505 and for providing 2-years postdoctoral fellowship for Dr D.B. Kaldybekov at the University of Reading. 506 The Chemical Analysis Facility (University of Reading) is thanked for access to a fluorescence 507 spectrophotometer and TEM. Dr S.K. Filippov acknowledges the financial support of U.S.-Czech 508 Republic Fulbright Commission. P.C. Turner Abattoirs (Farnborough, UK) is also acknowledged for 509 providing lamb urinary bladders for experiments.

\section{$510 \quad$ Conflict of interest}

511 The authors have no conflicts of interest to disclose.

\section{$512 \quad$ Author contributions}

513 The manuscript was written through contributions of all authors. All authors have given approval 514 to the final version of the manuscript.

\section{Appendix A. Supplementary material}

516 Supplementary data associated with this article is available free of charge. It contains a standard 517 curve used to determine the amount of encapsulated fluorescein in the PLGA-PEG nanoparticles (Fig. 518 S1); detailed schematic illustration of SMIT test (Fig. S2) and the photographs of mucus production by 519 Arion lusitanicus slugs in contact with test materials (Fig. S3). 
$\mathrm{AU}$, artificial urine; $\mathrm{BAC}$, benzalkonium chloride; $\mathrm{BC}$, bladder cancer; $\mathrm{D}_{2} \mathrm{O}$, deuterium oxide;

522 DLS, dynamic light scattering; DMSO, dimethyl sulfoxide; EE\%, encapsulation efficiency; IDD, 523 intravesical drug delivery; LC\%, loading capacity; Mal, maleimide; MHA, 6-Maleimidohexanoic acid; 524 MP\%, mucus production; NPs, nanoparticles; PBS, phosphate buffered saline; PDI, polydispersity index; 525 PEG, poly(ethylene glycol) methyl ether; PEG-Mal, methoxypolyethylene glycol maleimide; PLGA526 PEG, poly(lactide-co-glycolide)-block-polyethylene glycol); SANS, small-angle neutron scattering; 527 SMIT, slug mucosal irritation test; TEM, transmission electron microscopy; UA, uranyl acetate; $\mathrm{WO}_{50}$, $528 \quad$ Wash Out 50\%.

\section{$529 \quad$ ORCID ID of authors}

530 Daulet B. Kaldybekov: https://orcid.org/0000-0002-7191-5465

531 Vitaliy V. Khutoryanskiy: https://orcid.org/0000-0002-7221-2630

\section{$532 \quad$ References}

533 [1] V. V. Khutoryanskiy, Mucoadhesive materials and drug delivery systems, John Wiley \& Sons, $534 \quad$ Ltd, Chichester, UK, 2014. doi:10.1002/9781118794203.

535 [2] G. Prosperi-Porta, S. Kedzior, B. Muirhead, H. Sheardown, Phenylboronic-acid-based polymeric micelles for mucoadhesive anterior segment ocular drug delivery, Biomacromolecules. 17 (2016) 1449-1457. doi:10.1021/acs.biomac.6b00054.

[4] Y.G. Seo, D.W. Kim, W.H. Yeo, T. Ramasamy, Y.K. Oh, Y.J. Park, J.A. Kim, D.H. Oh, S.K. Ku, 
nanomicelles as a rectal drug delivery system for enhanced chemotherapeutic effect, Pharm. Res. 30 (2013) 1860-1870. doi:10.1007/s11095-013-1029-0.

[5] C.M. Caramella, S. Rossi, F. Ferrari, M.C. Bonferoni, G. Sandri, Mucoadhesive and thermogelling systems for vaginal drug delivery, Adv. Drug Deliv. Rev. 92 (2015) 39-52. doi:10.1016/j.addr.2015.02.001.

[6] C. Rieger, D. Kunhardt, A. Kaufmann, D. Schendel, D. Huebner, K. Erdmann, S. Propping, M.P.

Wirth, B. Schwenzer, S. Fuessel, S. Hampel, Characterization of different carbon nanotubes for the development of a mucoadhesive drug delivery system for intravesical treatment of bladder cancer, Int. J. Pharm. 479 (2015) 357-363. doi:10.1016/j.ijpharm.2015.01.017.

[7] M. Hornof, W. Weyenberg, A. Ludwig, A. Bernkop-Schnürch, Mucoadhesive ocular insert based 553 on thiolated poly(acrylic acid): Development and in vivo evaluation in humans, J. Control. Release. 89 (2003) 419-428. doi:10.1016/S0168-3659(03)00135-4.

[8] J.F. Fangueiro, F. Veiga, A.M. Silva, E.B. Souto, Ocular drug delivery - new strategies for targeting anterior and posterior segments of the eye, Curr. Pharm. Des. 22 (2016) 1135-1146. doi:http://dx.doi.org/10.2174/1381612822666151216145900.

[9] M. Kapoor, J.C. Cloyd, R.A. Siegel, A review of intranasal formulations for the treatment of 559 seizure emergencies, J. Control. Release. 237 (2016) 147-159. doi:10.1016/j.jconrel.2016.07.001.

[10] L. Kozlovskaya, M. Abou-Kaoud, D. Stepensky, Quantitative analysis of drug delivery to the brain

[11] C. Mugabe, Y. Matsui, A.I. So, M.E. Gleave, J.H.E. Baker, A.I. Minchinton, I. Manisali, R. 563 564 Liggins, D.E. Brooks, H.M. Burt, In vivo evaluation of mucoadhesive nanoparticulate docetaxel for intravesical treatment of non-muscle-invasive bladder cancer, Clin. Cancer Res. 17 (2011) 2788-2798. doi:10.1158/1078-0432.CCR-10-2981.

[12] R.R. De Araújo Pereira, M.L. Bruschi, Vaginal mucoadhesive drug delivery systems, Drug Dev. 
[13] O.M. Kolawole, W.M. Lau, H. Mostafid, V. V. Khutoryanskiy, Advances in intravesical drug delivery systems to treat bladder cancer, Int. J. Pharm. 532 (2017) 105-117. doi:10.1016/J.IJPHARM.2017.08.120.

[14] A.R. Mackie, F.M. Goycoolea, B. Menchicchi, C.M. Caramella, F. Saporito, S. Lee, K. Stephansen, I.S. Chronakis, M. Hiorth, M. Adamczak, M. Waldner, H. Mørck Nielsen, L. Marcelloni, Innovative methods and applications in mucoadhesion research, Macromol. Biosci. 17 (2017) 1600534. doi:10.1002/mabi.201600534.

[15] P. Tyagi, P.C. Wu, M. Chancellor, N. Yoshimura, L. Huang, Recent advances in intravesical 576

[16] S. GuhaSarkar, R. Banerjee, Intravesical drug delivery: Challenges, current status, opportunities and novel strategies, J. Control. Release. 148 (2010) 147-159. doi:10.1016/j.jconrel.2010.08.031.

[17] I.A. Sogias, A.C. Williams, V. V. Khutoryanskiy, Why is chitosan mucoadhesive?, Biomacromolecules. 9 (2008) 1837-1842. doi:10.1021/bm800276d.

[18] E. Bilensoy, C. Sarisozen, G. Esendağli, A.L. Doğan, Y. Aktaş, M. Şen, N.A. Mungan, Intravesical cationic nanoparticles of chitosan and polycaprolactone for the delivery of Mitomycin C to bladder tumors, Int. J. Pharm. 371 (2009) 170-176. doi:10.1016/j.ijpharm.2008.12.015.

[19] H.T. Ta, C.R. Dass, D.E. Dunstan, Injectable chitosan hydrogels for localised cancer therapy, J. Control. Release. 126 (2008) 205-216. doi:10.1016/j.jconrel.2007.11.018.

[20] I. Bravo-Osuna, M. Noiray, E. Briand, A.M. Woodward, P. Argüeso, I.T.M. Martínez, R. HerreroVanrell, G. Ponchel, Interfacial interaction between transmembrane ocular mucins and adhesive polymers and dendrimers analyzed by surface plasmon resonance, Pharm. Res. 29 (2012) 2329_ 2340. doi:10.1007/s11095-012-0761-1.

[21] M. Burjak, M. Bogataj, M. Velnar, I. Grabnar, A. Mrhar, The study of drug release from microspheres adhered on pig vesical mucosa, Int. J. Pharm. 224 (2001) 123-130. doi:10.1016/S0378-5173(01)00748-7. 
[22] A. Bernkop-Schnürch, Thiomers: The next generation of mucoadhesive polymers, Adv. Drug Deliv. Rev. 57 (2005) 1569-1582. doi:10.1016/j.addr.2005.07.002.

[23] A. Bernkop-Schnürch, D. Guggi, Y. Pinter, Thiolated chitosans: Development and in vitro evaluation of a mucoadhesive, permeation enhancing oral drug delivery system, J. Control. Release. 94 (2004) 177-186. doi:10.1016/j.jconrel.2003.10.005.

[24] T. Schmitz, V. Grabovac, T.F. Palmberger, M.H. Hoffer, A. Bernkop-Schnürch, Synthesis and characterization of a chitosan-N-acetyl cysteine conjugate, Int. J. Pharm. 347 (2008) 79-85. doi:10.1016/j.ijpharm.2007.06.040.

[25] J. Iqbal, G. Shahnaz, S. Dünnhaupt, C. Müller, F. Hintzen, A. Bernkop-Schnürch, Preactivated thiomers as mucoadhesive polymers for drug delivery, Biomaterials. 33 (2012) 1528-1535. doi:10.1016/j.biomaterials.2011.10.021.

[26] J. Barthelmes, G. Perera, J. Hombach, S. Dünnhaupt, A. Bernkop-Schnürch, Development of a mucoadhesive nanoparticulate drug delivery system for a targeted drug release in the bladder, Int. J. Pharm. 416 (2011) 339-345. doi:10.1016/j.ijpharm.2011.06.033.

[27] N. Denora, A. Lopedota, M. Perrone, V. Laquintana, R.M. Iacobazzi, A. Milella, E. Fanizza, N. Depalo, A. Cutrignelli, A. Lopalco, M. Franco, Spray-dried mucoadhesives for intravesical drug delivery using N-acetylcysteine- and glutathione-glycol chitosan conjugates, Acta Biomater. 43 (2016) 170-184. doi:10.1016/j.actbio.2016.07.025.

[28] P. Tonglairoum, R.P. Brannigan, P. Opanasopit, V. V. Khutoryanskiy, Maleimide-bearing nanogels as novel mucoadhesive materials for drug delivery, J. Mater. Chem. B. 4 (2016) 65816587. doi:10.1039/C6TB02124G.

[29] D.B. Kaldybekov, P. Tonglairoum, P. Opanasopit, V. V. Khutoryanskiy, Mucoadhesive maleimide-functionalised liposomes for drug delivery to urinary bladder, Eur. J. Pharm. Sci. 111 (2018) 83-90. doi:10.1016/j.ejps.2017.09.039. 
terminated PEG as drug carriers with enhanced mucoadhesion, Carbohydr. Polym. 175 (2017) 337-346. doi:10.1016/j.carbpol.2017.07.076.

[31] N. Sahatsapan, T. Rojanarata, T. Ngawhirunpat, P. Opanasopit, P. Tonglairoum, 6Maleimidohexanoic acid-grafted chitosan: A new generation mucoadhesive polymer, Carbohydr. Polym. 202 (2018) 258-264. doi:https://doi.org/10.1016/j.carbpol.2018.08.119.

[32] Y. Xu, C.S. Kim, D.M. Saylor, D. Koo, Polymer degradation and drug delivery in PLGA-based drug-polymer applications: A review of experiments and theories, J. Biomed. Mater. Res. - Part B Appl. Biomater. 105 (2017) 1692-1716. doi:10.1002/jbm.b.33648.

[34] K. Zhang, X. Tang, J. Zhang, W. Lu, X. Lin, Y. Zhang, B. Tian, H. Yang, H. He, PEG-PLGA therapeutic effect of DOX-PLGA-PEG micelles decorated with bivalent fragment HAb18 $\mathrm{F}\left(\mathrm{ab}^{\prime}\right) 2$ for hepatocellular carcinoma, Biomacromolecules. $11 \quad$ (2010) 2422-2431. doi:10.1021/bm1005992. copolymers: Their structure and structure-influenced drug delivery applications, J. Control. Release. 183 (2014) 77-86. doi:10.1016/j.jconrel.2014.03.026.

35] U. Bazylińska, Rationally designed double emulsion process for co-encapsulation of hybrid cargo in stealth nanocarriers, Colloids Surfaces A Physicochem. Eng. Asp. 532 (2017) 476-482. doi:10.1016/j.colsurfa.2017.04.027.

[36] Y. Pei, M.F. Mohamed, M.N. Seleem, Y. Yeo, Particle engineering for intracellular delivery of vancomycin to methicillin-resistant Staphylococcus aureus (MRSA)-infected macrophages, J. Control. Release. 267 (2017) 133-143. doi:10.1016/j.jconrel.2017.08.007.

[37] A.S. Wadajkar, J.G. Dancy, N.B. Roberts, N.P. Connolly, D.K. Strickland, J.A. Winkles, G.F. Woodworth, A.J. Kim, Decreased non-specific adhesivity, receptor targeted (DART) nanoparticles exhibit improved dispersion, cellular uptake, and tumor retention in invasive gliomas, J. Control. Release. 267 (2017) 144-153. doi:10.1016/j.jconrel.2017.09.006. 
643 [38] L. Zhang, Y. Shi, Y. Song, X. Sun, X. Zhang, K. Sun, Y. Li, The use of low molecular weight 644 protamine to enhance oral absorption of exenatide, Int. J. Pharm. 547 (2018) $265-273$. 645 doi:https://doi.org/10.1016/j.ijpharm.2018.05.055.

[39] B. Zhang, P. Sai Lung, S. Zhao, Z. Chu, W. Chrzanowski, Q. Li, Shape dependent cytotoxicity of PLGA-PEG nanoparticles on human cells, Sci. Rep. 7 (2017) 7315. doi:10.1038/s41598-01707588-9.

[40] A. Radulescu, N.K. Szekely, M.-S. Appavou, KWS-2: Small angle scattering diffractometer, J. 650 Large-Scale Res. Facil. JLSRF. 1 (2015) A29. doi:10.17815/jlsrf-1-27.

[41] I. Breßler, J. Kohlbrecher, A.F. Thünemann, SASfit: A tool for small-angle scattering data 652 analysis using a library of analytical expressions, J. Appl. Crystallogr. 48 (2015) 1587-1598. doi:10.1107/S1600576715016544.

[42] J.S. Pedersen, M.C. Gerstenberg, The structure of P85 Pluronic block copolymer micelles determined by small-angle neutron scattering, Colloids Surfaces A Physicochem. Eng. Asp. 213 (2003) 175-187. doi:10.1016/S0927-7757(02)00511-3.

O. V. Khutoryanskaya, P.W.J. Morrison, S.K. Seilkhanov, M.N. Mussin, E.K. Ozhmukhametova, T.K. Rakhypbekov, V. V. Khutoryanskiy, Hydrogen-bonded complexes and blends of poly(acrylic acid) and methylcellulose: Nanoparticles and mucoadhesive films for ocular delivery of riboflavin, Macromol. Biosci. 14 (2014) 225-234. doi:10.1002/mabi.201300313.

[44] S. Chutipongtanate, V. Thongboonkerd, Systematic comparisons of artificial urine formulas for in vitro cellular study, Anal. Biochem. 402 (2010) 110-112. doi:10.1016/j.ab.2010.03.031.

[46] A.I. Vogel, B.S. Furniss, Vogel's textbook of practical organic chemistry, Longman, Harlow, 667 Essex, England, 1989. https://books.google.kz/books?id=2eQPAQAAMAAJ. 
[47] B. Yang, J.P. Lowe, R. Schweins, K.J. Edler, Small angle neutron scattering studies on the internal structure of poly(lactide-co-glycolide)-block-poly(ethylene glycol) nanoparticles as drug delivery vehicles, Biomacromolecules. 16 (2015) 457-464. doi:10.1021/bm501519u.

[48] J.H. Lass, Advances in Corneal Research: Selected Transactions of the World Congress on the 672 Cornea IV, Springer Science \& Business Media, Boston, MA, 1998. https://books.google.kz/books?id=7wGH80U8tUIC.

[50] W. Burchard, Static and dynamic light scattering from branched polymers and biopolymers, Light Scatt. from Polym. Adv. Polym. Sci. 48 (1983) 1-124. doi:10.1007/3-540-12030-0_1. 1996. https://books.google.co.uk/books?id=RsNQGIlEbpwC.

D. Gromadzki, S. Filippov, M. Netopilík, R. Makuška, A. Jigounov, J. Pleštil, J. Horský, P. Štěpánek, Combination of "living" nitroxide-mediated and photoiniferter-induced "grafting from" free-radical polymerizations: From branched copolymers to unimolecular micelles and microgels, Eur. Polym. J. 45 (2009) 1748-1758. doi:10.1016/j.eurpolymj.2009.02.022.

[53] M. Hruby, C. Konak, J. Kucka, M. Vetrik, S.K. Filippov, D. Vetvicka, H. Mackova, G. Karlsson, K. Edwards, B. Rihova, K. Ulbrich, Thermoresponsive, hydrolytically degradable polymer micelles intended for radionuclide delivery, Macromol. Biosci. 9 (2009) 1016-1027. doi:10.1002/mabi.200900083.

[54] T. Riley, C.R. Heald, S. Stolnik, M.C. Garnett, L. Illum, S.S. Davis, S.M. King, R.K. Heenan, S.C. Purkiss, R.J. Barlow, P.R. Gellert, C. Washington, Core-shell structure of PLA-PEG nanoparticles used for drug delivery, Langmuir. 19 (2003) 8428-8435. doi:10.1021/la020911h.

[55] M.J. Park, K. Char, Gelation of PEO-PLGA-PEO triblock copolymers induced by macroscopic phase separation, Langmuir. 20 (2004) 2456-2465. doi:10.1021/la035573e. 
693 [56] N.K. Khorshid, K. Zhu, K.D. Knudsen, S. Bekhradnia, S.A. Sande, B. Nyström, Novel structural 694 changes during temperature-induced self-assembling and gelation of PLGA-PEG-PLGA triblock 695 copolymer in aqueous solutions, Macromol. Biosci. $16 \quad$ (2016) 1838-1852. 696 doi:10.1002/mabi.201600277.

697 [57] T. Maeda, M. Kitagawa, A. Hotta, S. Koizumi, Thermo-responsive nanocomposite hydrogels 698 based on PEG-b-PLGA diblock copolymer and laponite, Polymers (Basel). 11 (2019) 250. 699 doi:10.3390/polym11020250.

700 [58] E. Adriaens, J.P. Remon, Gastropods as an evaluation tool for screening the irritating potency of 701 absorption enhancers and drugs, Pharm. Res. $16 \quad$ (1999) 1240-1244. 702 doi:10.1023/A:1014801714590.

703 [59] E. Adriaens, K. Dierckens, T.G.M. Bauters, H.J. Nelis, F. Van Goethem, P. Vanparys, J.P. Remon, 704 The mucosal toxicity of different benzalkonium chloride analogues evaluated with an alternative 705 test using slugs, Pharm. Res. 18 (2001) 937-942. doi:10.1023/A:1010928025753.

706 [60] J. Lenoir, C. Bachert, J.P. Remon, E. Adriaens, The Slug Mucosal Irritation (SMI) assay: A tool 707 for the evaluation of nasal discomfort, Toxicol. Vitr. 27 (2013) $1954-1961$. $708 \quad$ doi:10.1016/j.tiv.2013.06.018.

709 [61] O. V. Khutoryanskaya, Z.A. Mayeva, G.A. Mun, V. V. Khutoryanskiy, Designing temperature710 responsive biocompatible copolymers and hydrogels based on 2-hydroxyethyl(meth)acrylates, 711 Biomacromolecules. 9 (2008) 3353-3361. doi:10.1021/bm8006242. 


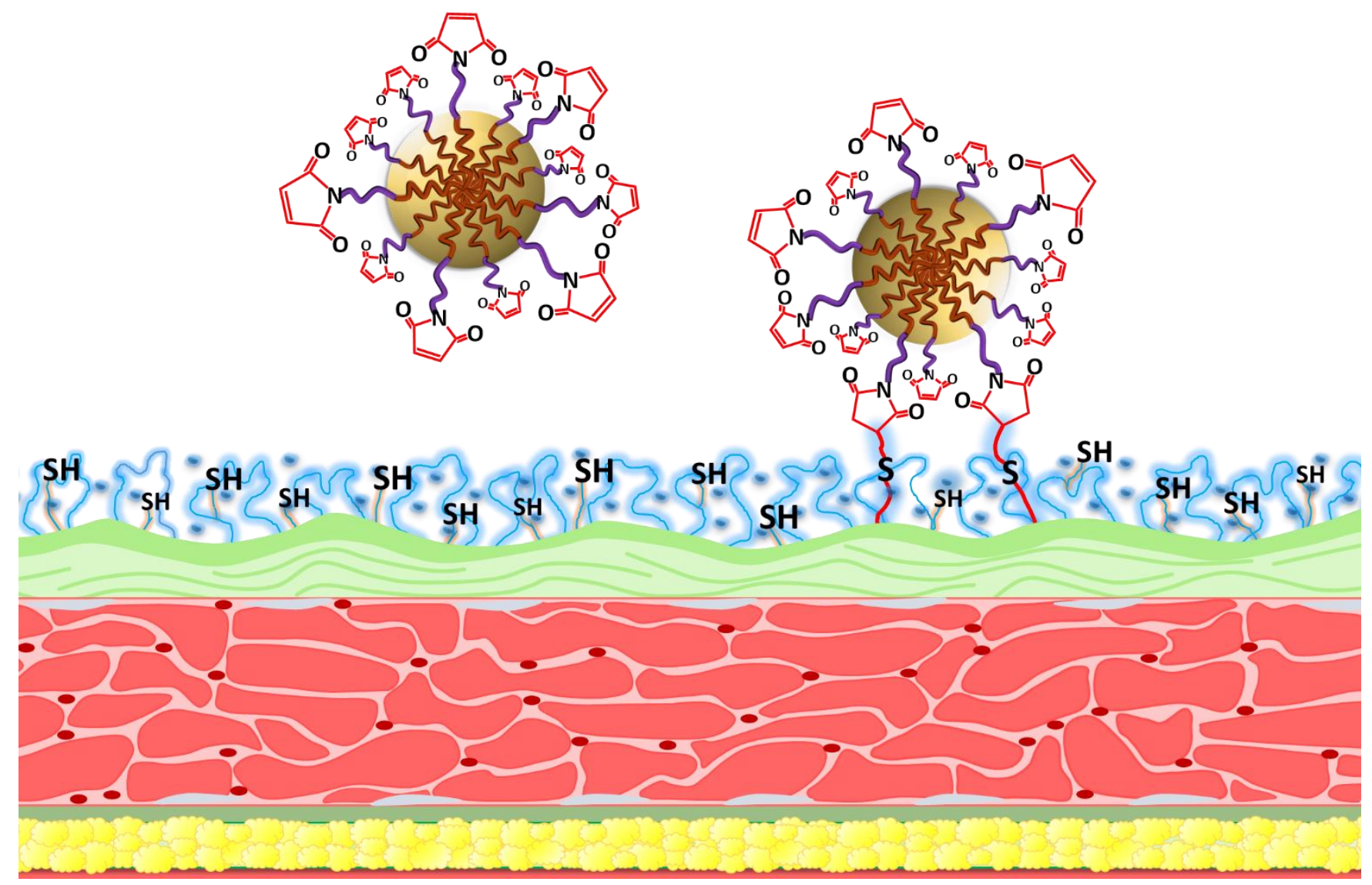

715 Schematic illustration depicting the mechanism of enhanced mucoadhesion of PLGA-PEG-Mal 716 nanoparticles on urinary bladder mucosa. 


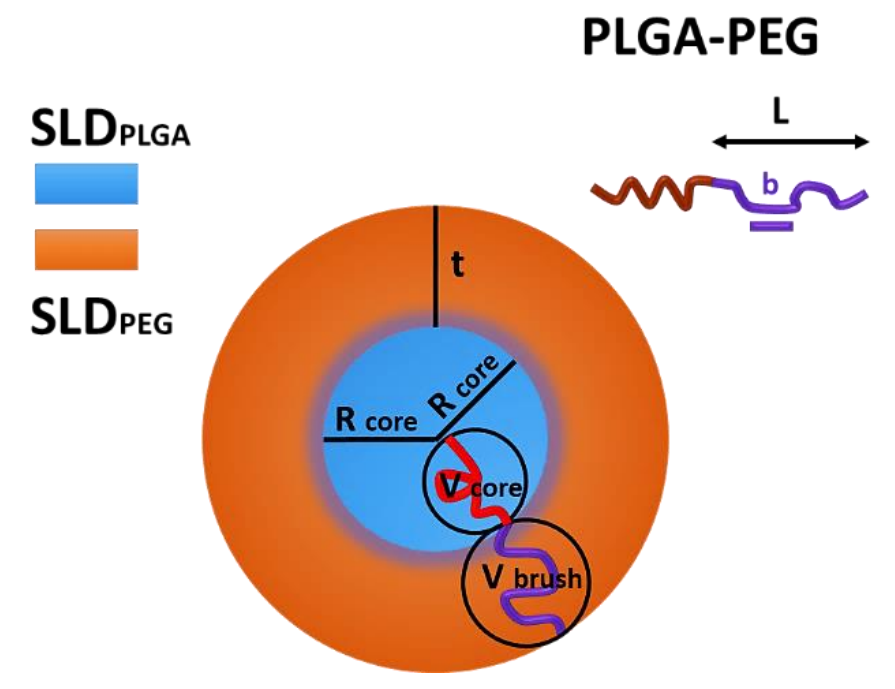

721 Fig. 1. The model used for the fitting of experimental SANS data.

722 (single column fitting image) 

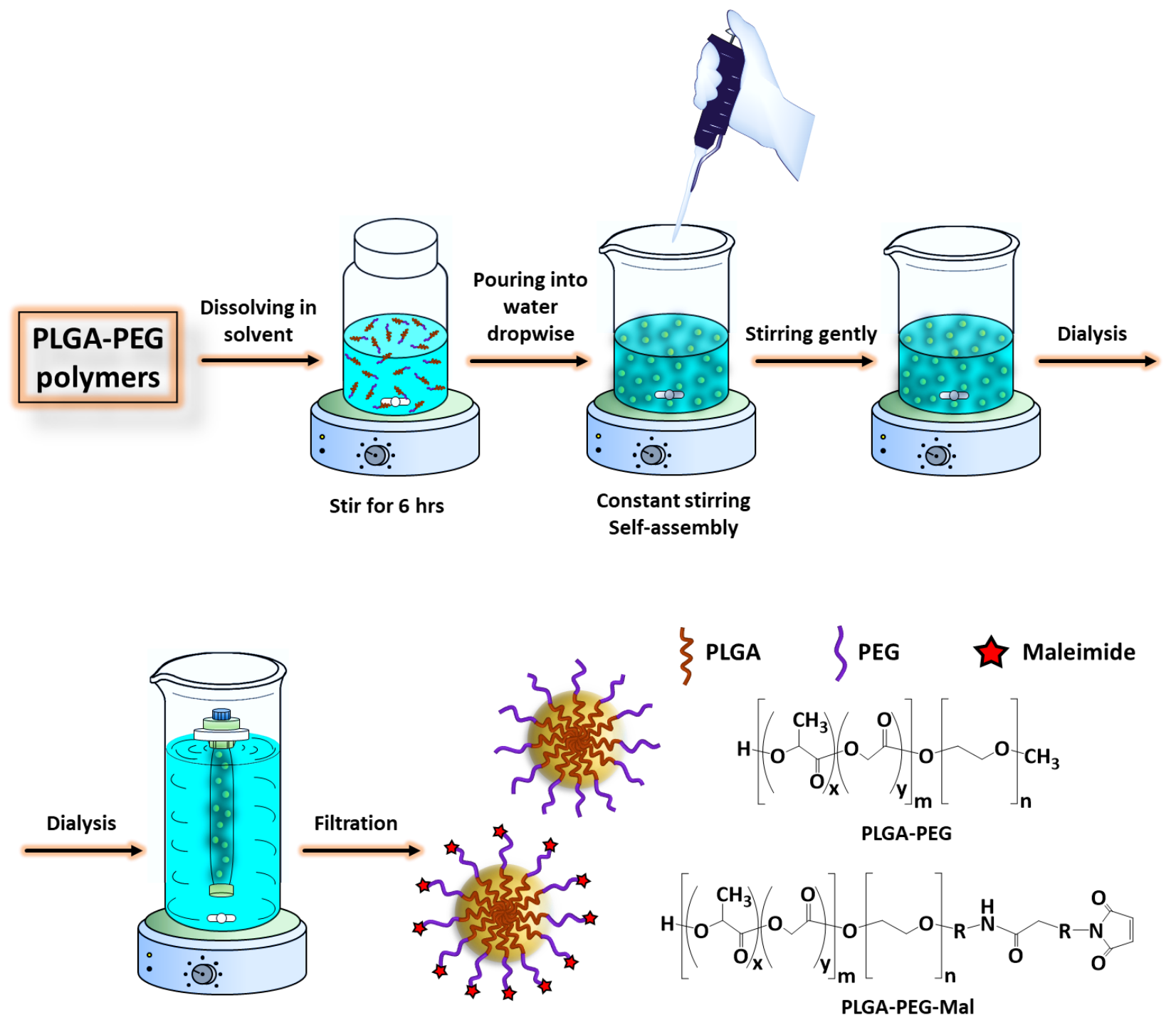

\section{PLGA-PEG nanoparticles}

724 Fig. 2. Illustrative diagram depicting the preparation of PLGA-PEG nanoparticles with maleimide725 functionalised surface.

726 (2-column fitting image) 

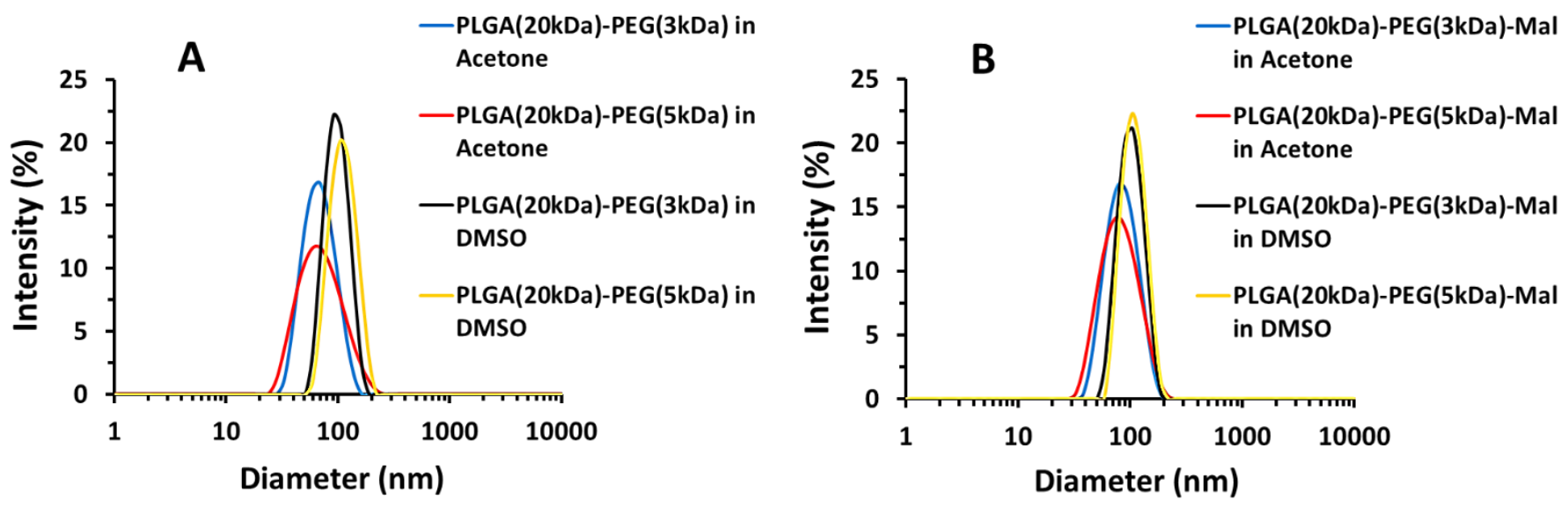

728 Fig. 3. Size distribution of PLGA-PEG (A) and PLGA-PEG-Mal (B) nanoparticles dissolved and 729 precipitated from acetone and DMSO as determined by DLS.

$730 \quad$ (2-column fitting image) 

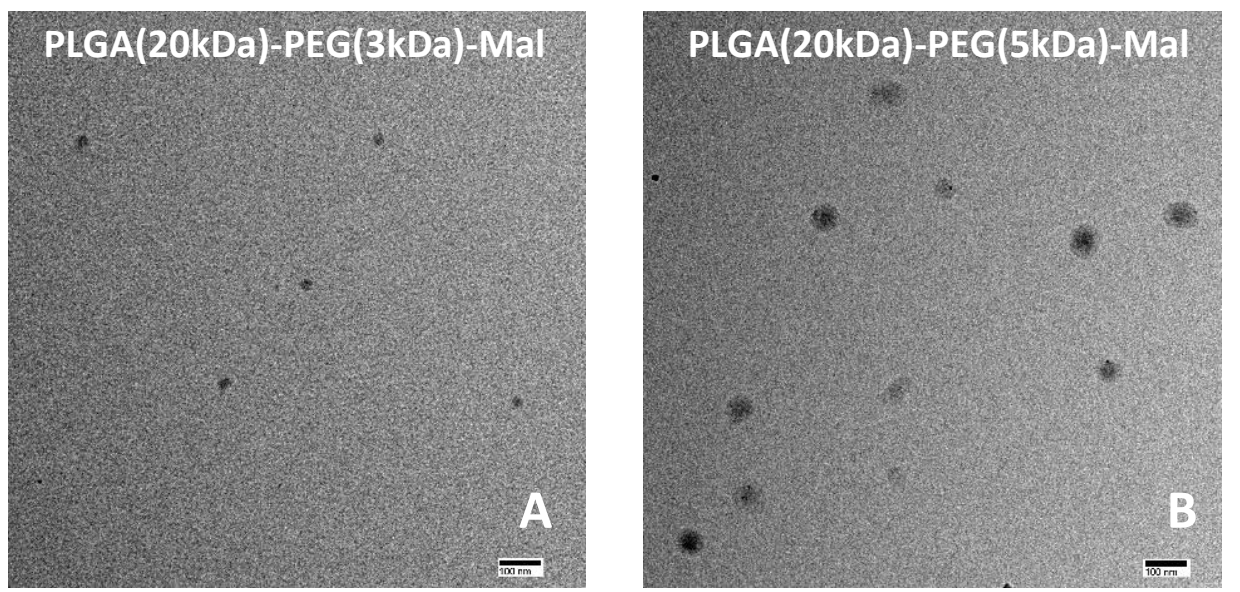

732 Fig. 4. TEM microphotographs of PLGA-PEG-Mal NPs dissolved and precipitated from acetone (A) and 733 DMSO (B). Scale bars are $100 \mathrm{~nm}$.

734 (single column fitting image) 

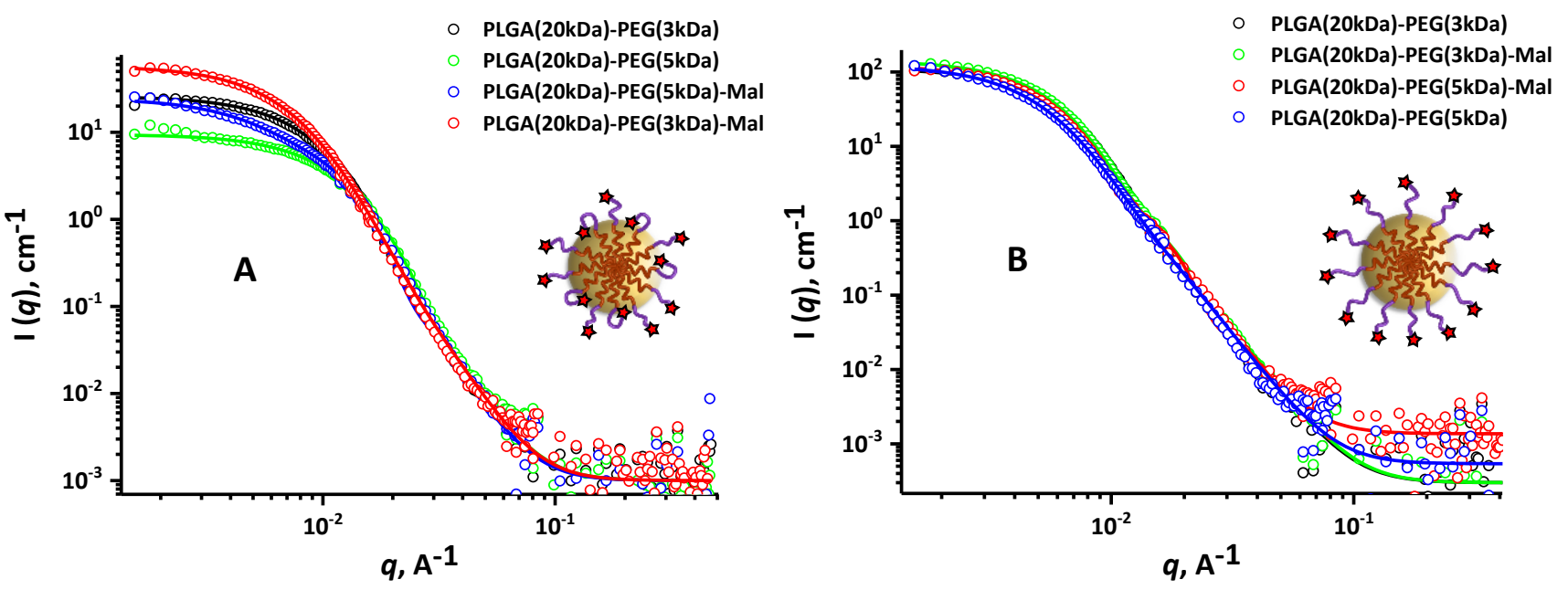

736 Fig. 5. SANS curves for PLGA-PEG nanoparticles dissolved and precipitated from acetone (A) and 737 DMSO (B). Solid line is a fitting curve. Inserts: Proposed structures of nanoparticles.

$738 \quad$ (2-column fitting image) 


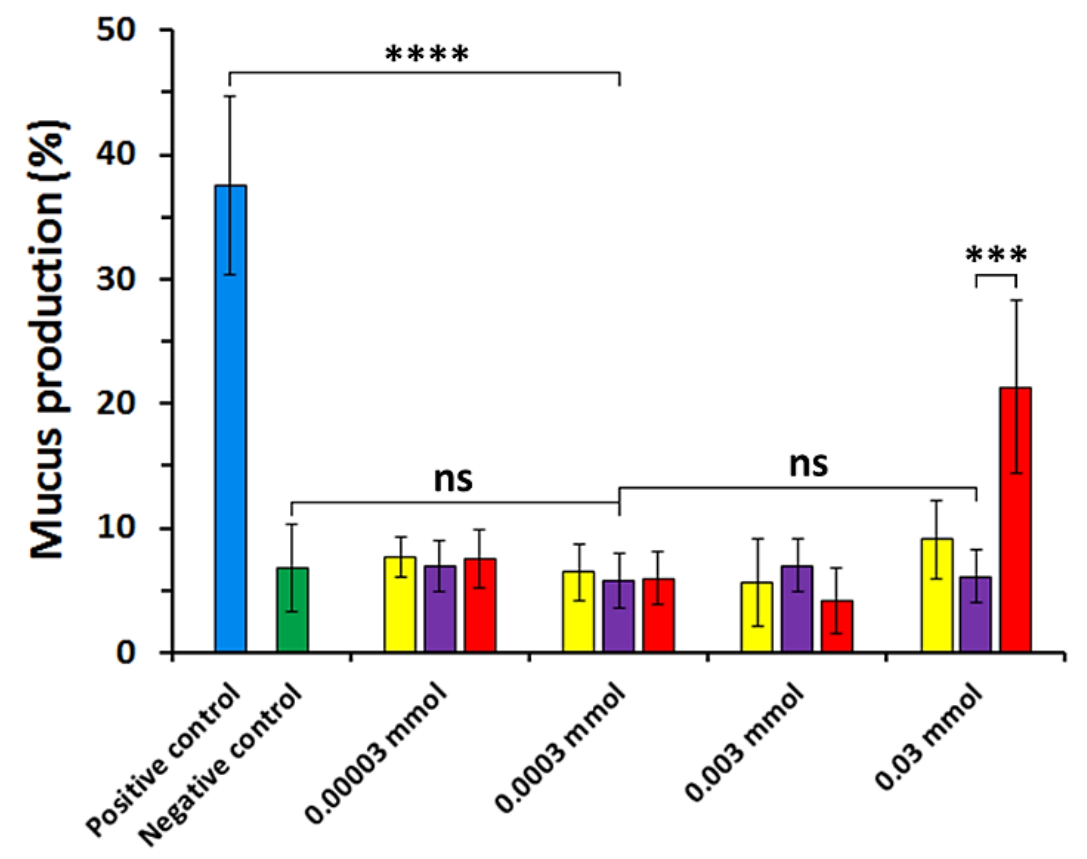

740 Fig. 6. Mucus production by Arion lusitanicus slugs in response to 60 min exposure to PEG, PEG-Mal 741 and MHA as well as positive (benzalkonium chloride - BAC) and negative (phosphate buffered saline 742 PBS) controls. Data are expressed as mean \pm standard deviation $(n=7)$. Statistically significant 743 differences are given as: $* * * *-p<0.0001 ; * * *-p<0.001$; ns - no significance.

$744 \quad$ (single column fitting image) 
PLGA(20kDa)-PEG(5kDa)-Mal
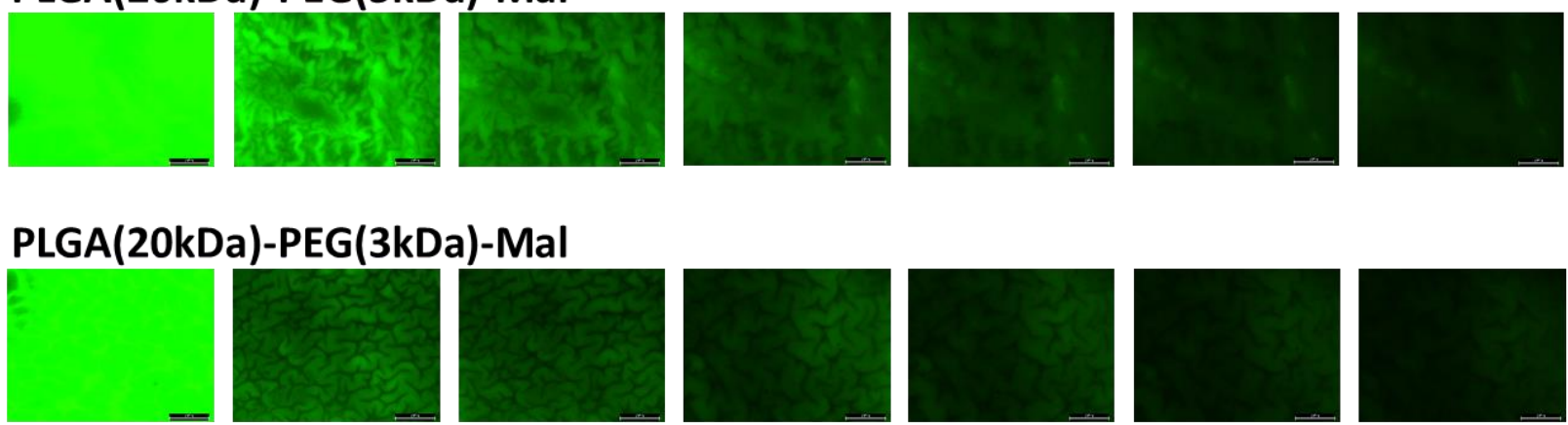

\section{PLGA(20kDa)-PEG(5kDa)}
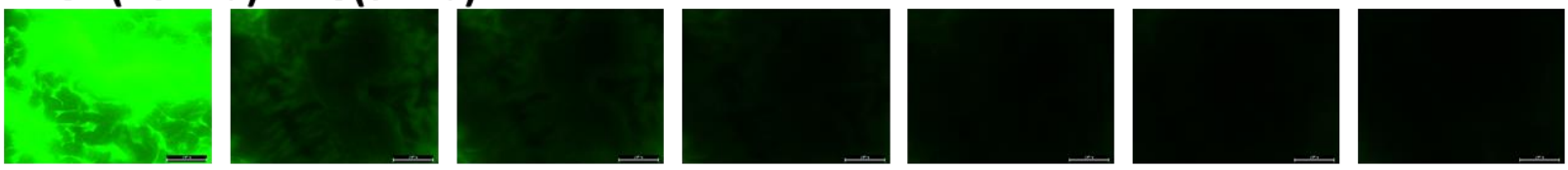

\section{PLGA(20kDa)-PEG(3kDa)}
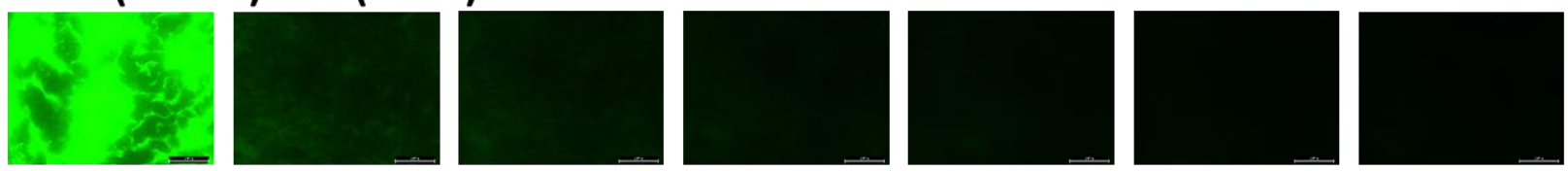

0

10

20

40

60

80

100

746 Fig. 7. Exemplar fluorescence images showing retention of PLGA-PEG nanoparticles on lamb urinary

747 bladder mucosa washed with different volumes of AU. Scale bars are $2 \mathrm{~mm}$.

$748 \quad$ (2-column fitting image) 


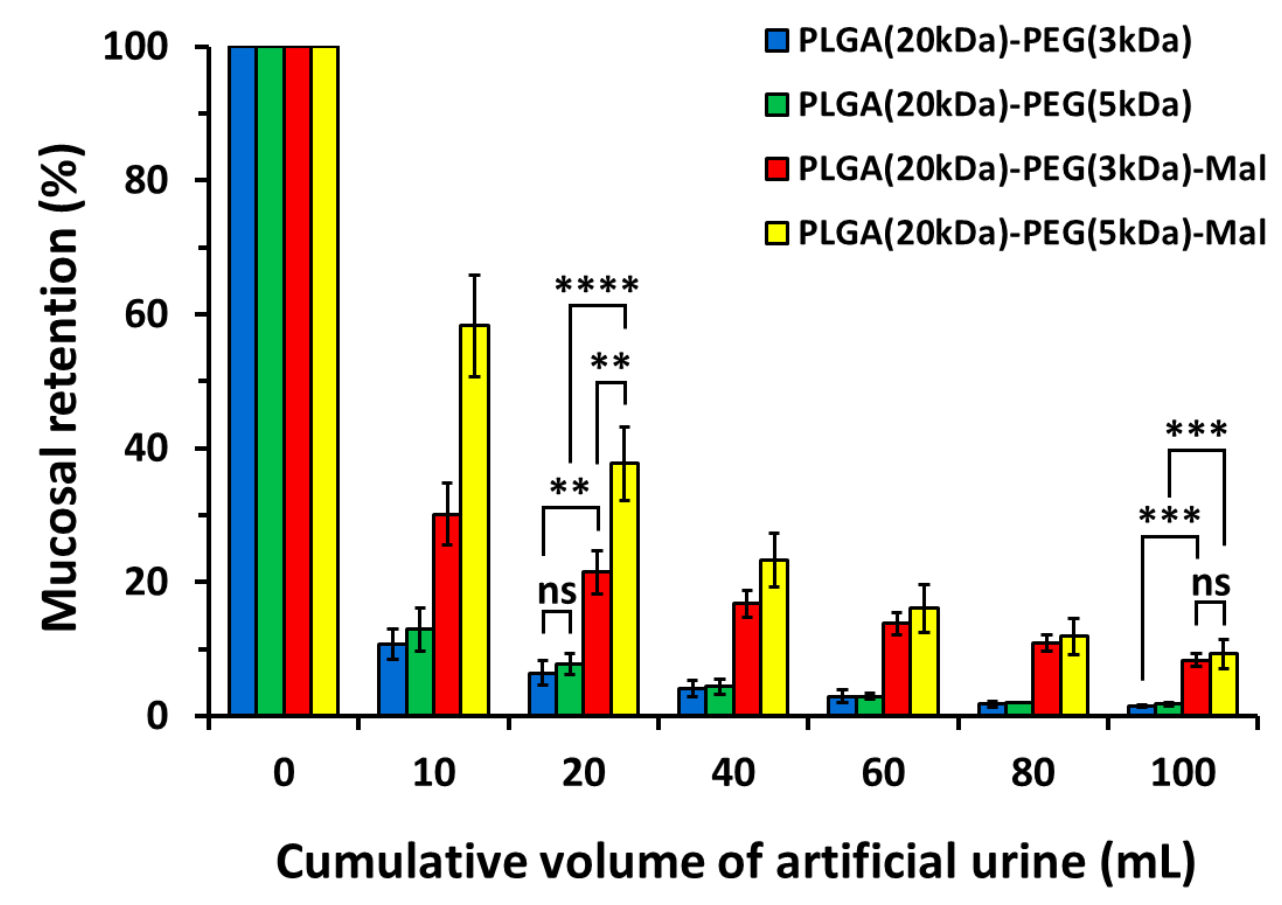

750 Fig. 8. Percentage retention of PLGA-PEG nanoparticles on lamb urinary bladder mucosa after irrigating 751 with different volumes of AU solution. Data are expressed as mean \pm standard deviation $(\mathrm{n}=3)$.

752 Statistically significant differences are given as: $* * *-p<0.0001 ; * * *-p<0.001 ; * *-p<0.01$; ns $753-$ no significance.

$754 \quad$ (single column fitting image) 


\section{TABLES WITH CAPTIONS}

756 Table 1. Physicochemical characteristics of PLGA-PEG nanoparticles.

\begin{tabular}{|c|c|c|c|c|c|c|c|}
\hline Formulation & $\begin{array}{l}\text { Solvent } \\
\text { used in NPs } \\
\text { preparation }\end{array}$ & $\begin{array}{l}\text { Mean } \\
\text { diameter } \\
(\mathrm{nm})\end{array}$ & PDI & $\begin{array}{l}\text { Zeta- } \\
\text { potential } \\
(\mathrm{mV})\end{array}$ & $\mathrm{EE} \%$ & $\mathrm{LC} \%$ & $\mathrm{WO}_{50}(\mathrm{~mL})^{*}$ \\
\hline $\mathrm{PLGA}_{20 \mathrm{~K}}-\mathrm{PEG}_{3 \mathrm{~K}}$ & & $94 \pm 1$ & 0.048 & $-20.8 \pm 0.5$ & $62.7 \pm 3.6$ & $3.1 \pm 0.2$ & $4 ;\left(\mathrm{R}^{2}=0.9860\right)$ \\
\hline $\mathrm{PLGA}_{20 \mathrm{~K}}-\mathrm{PEG}_{5 \mathrm{~K}}$ & DMSO & $105 \pm 1$ & 0.070 & $-21.6 \pm 0.4$ & $63.8 \pm 6.9$ & $3.2 \pm 0.4$ & $5 ;\left(\mathrm{R}^{2}=0.9872\right)$ \\
\hline $\mathrm{PLGA}_{20 \mathrm{~K}}-\mathrm{PEG}_{3 \mathrm{~K}}-\mathrm{Mal}$ & & $98 \pm 1$ & 0.060 & $-17.7 \pm 0.6$ & $60.0 \pm 4.2$ & $3.0 \pm 0.2$ & $6 ;\left(\mathrm{R}^{2}=0.9931\right)$ \\
\hline $\mathrm{PLGA}_{20 \mathrm{~K}-\mathrm{PEG}}{ }_{5 \mathrm{~K}-\mathrm{Mal}}$ & & $104 \pm 1$ & 0.067 & $-12.2 \pm 0.5$ & $55.0 \pm 5.8$ & $3.0 \pm 0.3$ & $15 ;\left(\mathrm{R}^{2}=1\right)$ \\
\hline $\mathrm{PLGA}_{20 \mathrm{~K}}-\mathrm{PEG}_{3 \mathrm{~K}}$ & & $64 \pm 1$ & 0.110 & $-8.1 \pm 0.7$ & N/A & N/A & N/A \\
\hline PLGA $20 K-P E G 5 K$ & Acetone & $68 \pm 1$ & 0.248 & $-5.2 \pm 0.6$ & N/A & N/A & N/A \\
\hline $\mathrm{PLGA}_{20 \mathrm{~K}}-\mathrm{PEG}_{3 \mathrm{~K}}-\mathrm{Mal}$ & & $80 \pm 1$ & 0.094 & $-16.5 \pm 0.6$ & N/A & N/A & N/A \\
\hline $\mathrm{PLGA}_{20 \mathrm{~K}-\mathrm{PEG}}{ }_{5 \mathrm{~K}}-\mathrm{Mal}$ & & $81 \pm 1$ & 0.206 & $-10.8 \pm 0.8$ & N/A & N/A & N/A \\
\hline
\end{tabular}

757 DMSO, dimethyl sulfoxide; PLGA-PEG, poly(lactide-co-glycolide)-block-polyethylene glycol); Mal, maleimide; PDI, polydispersity 758 index; EE\%, encapsulation efficiency; $\mathrm{LC} \%$, loading capacity; $\mathrm{WO}_{50}$, Wash Out 50\% profile is a volume of artificial urine required to 759 wash out $50 \%$ liquid formulation; N/A, not applicable. *Polynomial fitting (5th order) was used to quantify $\mathrm{WO}_{50}$ values. Results are 760 given as mean \pm standard deviation $(\mathrm{n}=3)$.

$761 \quad$ (2-column fitting table) 
762 Table 2. Comparison of structural parameters obtained from samples PLGA-PEG nanoparticles in $\mathrm{D}_{2} \mathrm{O}$

\begin{tabular}{|c|c|c|c|c|c|c|}
\hline Formulation & $\begin{array}{l}\text { Solvent used in NPs } \\
\text { preparation }\end{array}$ & $R_{g}(\mathrm{~nm})$ & $R_{g} / R_{h}$ & $R_{\text {core }}(\mathrm{nm})$ & $\sigma$ & $R_{\text {gchain }}(\mathrm{nm})$ \\
\hline $\mathrm{PLGA}_{20 \mathrm{~K}}-\mathrm{PEG}_{3 \mathrm{~K}}$ & & $35.7 \pm 1.4$ & 0.76 & $14.8 \pm 0.1$ & 0.43 & $12.0 \pm 0.1$ \\
\hline $\mathrm{PLGA}_{20 \mathrm{~K}}-\mathrm{PEG}_{5 \mathrm{~K}}$ & & $40.8 \pm 1.5$ & 0.78 & $15.4 \pm 0.2$ & 0.63 & $12.1 \pm 0.3$ \\
\hline $\mathrm{PLGA}_{20 \mathrm{~K}}-\mathrm{PEG}_{3 \mathrm{~K}}-\mathrm{Mal}$ & DMSO & $35.7 \pm 0.9$ & 0.73 & $14.6 \pm 0.1$ & 0.31 & $14.4 \pm 0.2$ \\
\hline $\mathrm{PLGA}_{20 \mathrm{~K}}-\mathrm{PEG}_{5 \mathrm{~K}}-\mathrm{Mal}$ & & $35.5 \pm 0.7$ & 0.68 & $15.8 \pm 0.2$ & 0.20 & $16.7 \pm 0.3$ \\
\hline PLGA20K-PEG $3 \mathrm{~K}$ & & $21.0 \pm 0.8$ & 0.66 & $10.2 \pm 0.2$ & 0.33 & $6.9 \pm 0.1$ \\
\hline $\mathrm{PLGA}_{20 \mathrm{~K}-\mathrm{PEG} 5 \mathrm{~K}}$ & Acetone & $36.0 \pm 0.5$ & 1.06 & $12.0 \pm 0.2$ & 0.21 & $8.6 \pm 0.1$ \\
\hline $\mathrm{PLGA}_{20 \mathrm{~K}}-\mathrm{PEG}_{3 \mathrm{~K}}-\mathrm{Mal}$ & & $30.0 \pm 1.0$ & 0.75 & $12.8 \pm 0.1$ & 0.50 & $4.1 \pm 0.2$ \\
\hline $\mathrm{PLGA}_{20 \mathrm{~K}}-\mathrm{PEG}_{5 \mathrm{~K}}-\mathrm{Mal}$ & & $33.0 \pm 2.0$ & 0.81 & $12.0 \pm 0.1$ & 0.24 & $8.3 \pm 0.1$ \\
\hline
\end{tabular}

763 DMSO, dimethyl sulfoxide; PLGA-PEG, poly(lactide-co-glycolide)-block-polyethylene glycol); Mal, maleimide; $R_{g}$, overall gyration

764 radius of a nanoparticle; $R_{\text {core }}$, core radius; $\sigma$, polydispersity parameter; $R_{\text {gchain }}$, gyration radius of a polymer chain in the corona.

$765 \quad$ (single column fitting table) 